\title{
GAME REFINEMENT RELATIONS AND METRICS
}

\author{
LUCA DE ALFARO $^{a}$, RUPAK MAJUMDAR $^{b}, \operatorname{VISHWANATH~RAMAN~}^{c}$, \\ AND MARIËLLE STOELINGA ${ }^{d}$ \\ ${ }^{a}$ CE Department, University of California, Santa Cruz \\ e-mail address: luca@soe.ucsc.edu \\ ${ }^{b}$ Department of CS, University of California, Los Angeles \\ e-mail address: rupak@cs.ucla.edu \\ ${ }^{c}$ CS Department, University of California, Santa Cruz \\ e-mail address: vishwa@soe.ucsc.edu \\ ${ }^{d}$ Department of CS, University of Twente, The Netherlands \\ e-mail address: marielle@cs.utwente.nl
}

ABstract. We consider two-player games played over finite state spaces for an infinite number of rounds. At each state, the players simultaneously choose moves; the moves determine a successor state. It is often advantageous for players to choose probability distributions over moves, rather than single moves. Given a goal (e.g., "reach a target state"), the question of winning is thus a probabilistic one: "what is the maximal probability of winning from a given state?".

On these game structures, two fundamental notions are those of equivalences and metrics. Given a set of winning conditions, two states are equivalent if the players can win the same games with the same probability from both states. Metrics provide a bound on the difference in the probabilities of winning across states, capturing a quantitative notion of state "similarity".

We introduce equivalences and metrics for two-player game structures, and we show that they characterize the difference in probability of winning games whose goals are expressed in the quantitative $\mu$-calculus. The quantitative $\mu$-calculus can express a large set of goals, including reachability, safety, and $\omega$-regular properties. Thus, we claim that our relations and metrics provide the canonical extensions to games, of the classical notion of bisimulation for transition systems. We develop our results both for equivalences and metrics, which generalize bisimulation, and for asymmetrical versions, which generalize simulation.

1998 ACM Subject Classification: F.4.1, F.1.1.

Key words and phrases: game semantics, minimax theorem, metrics, $\omega$-regular properties, quantitative $\mu$-calculus, probabilistic choice, equivalence of states, refinement of states.

* A version of this paper titled "Game Relations and Metrics" appeared in the $22^{\text {nd }}$ Annual IEEE Symposium on Logic in Computer Science, July 2007.

\section{LOGICAL METHODS} IN COMPUTER SCIENCE
DOI:10.2168/LMCS-4 (3:7) 2008
(C) L. de Alfaro, R. Majumdar, V. Raman, and M. Stoelinga (C) Creative Commons 


\section{INTRODUCTION}

We consider two-player games played for an infinite number of rounds over finite state spaces. At each round, the players simultaneously and independently select moves; the moves then determine a probability distribution over successor states. These games, known variously as stochastic games [27] or concurrent games [5, 1, 7], generalize many common structures in computer science, from transition systems, to Markov chains [15] and Markov decision processes [8]. The games are turn-based if, at each state, at most one of the players has a choice of moves, and deterministic if the successor state is uniquely determined by the current state, and by the moves chosen by the players.

It is well-known that in such games with simultaneous moves it is often advantageous for the players to randomize their moves, so that at each round, they play not a single "pure" move, but rather, a probability distribution over the available moves. These probability distributions over moves, called mixed moves [23], lead to various notions of equilibria [32, 23], such as the equilibrium result expressed by the minimax theorem [32. Intuitively, the benefit of playing mixed, rather than pure, moves lies in preventing the adversary from tailoring a response to the individual move played. Even for simple reachability games, the use of mixed moves may allow players to win, with probability 1 , games that they would lose (i.e., win with probability 0) if restricted to playing pure moves [5]. With mixed moves, the question of winning a game with respect to a goal is thus a probabilistic one: what is the maximal probability a player can be guaranteed of winning, regardless of how the other player plays? This probability is known, in brief, as the winning probability.

In structures ranging from transition systems to Markov decision processes and games, a fundamental question is the one of equivalence of states. Given a suitably large class $\Phi$ of properties, containing all properties of interest to the modeler, two states are equivalent if the same properties hold in both states. For a property $\varphi$, denote the value of $\varphi$ at $s$ by $\varphi(s)$ : in the case of games, this might represent the maximal probability of a player winning with respect to a goal expressed by $\varphi$. Two states $s$ and $t$ are equivalent if $\varphi(s)=\varphi(t)$ for all $\varphi \in \Phi$. For (finite-branching) transition systems, and for the class of properties $\Phi$ expressible in the $\mu$-calculus [17], state equivalence is captured by bisimulation [22]; for Markov decision processes, it is captured by probabilistic bisimulation [25]. For quantitative properties, a notion related to equivalence is that of a metric: a metric provides a tight bound for how much the value of a property can differ at states of the system, and provides thus a quantitative notion of similarity between states. Given a set $\Phi$ of properties, the metric distance of two states $s$ and $t$ can be defined as $\sup _{\varphi \in \Phi}|\varphi(s)-\varphi(t)|$. Metrics for Markov decision processes have been studied in [9, 30, 31, 10, 11]. Obviously, the metrics and relations are connected, in the sense that the relations are the kernels of the metrics (the pairs of states having metric distance 0 ). The metrics and relations are at the heart of many verification techniques, from approximate reasoning (one can substitute states that are close in the metric) to system reductions (one can collapse equivalent states) to compositional reasoning and refinement (providing a notion of substitutivity of equivalents).

We introduce metrics and equivalence relations for concurrent games, with respect to the class of properties $\Phi$ expressible in the quantitative $\mu$-calculus [7, 21]. We claim that these metrics and relations represent the canonical extension of bisimulation to games. We also introduce asymmetrical versions of these metrics and equivalences, which constitute the canonical extension of simulation. 
An equivalence relation for deterministic games that are either turn-based, or where the players are constrained to playing pure moves, has been introduced in [2] and called alternating bisimulation. Relations and metrics for the general case of concurrent games have so far proved elusive, with some previous attempts at their definition by a subset of the authors following a subtly flawed approach [6, 19]. The cause of the difficulty goes to the heart of the definition of bisimulation. In the definition of bisimulation for transition systems, for every pair $s, t$ of bisimilar states, we require that if $s$ can go to a state $s^{\prime}$, then $t$ should be able to go to $t^{\prime}$, such that $s^{\prime}$ and $t^{\prime}$ are again bisimilar (we also ask that $s, t$ have an equivalent predicate valuation). This definition has been extended to Markov decision processes by requiring that for every mixed move from $s$, there is a mixed move from $t$, such that the moves induce probability distributions over successor states that are equivalent modulo the underlying bisimulation [25, 24]. Unfortunately, the generalization of this appealing definition to games fails. It turns out, as we prove in this paper, that requiring players to be able to replicate probability distributions over successors (modulo the underlying equivalence) leads to an equivalence that is too fine, and that may fail to relate states at which the same quantitative $\mu$-calculus formulas hold. We show that phrasing the definition in terms of distributions over successor states is the wrong approach for games; rather, the definition should be phrased in terms of expectations of certain metric-bounded quantities.

Our starting point is a closer look at the definition of metrics for Markov decision processes. We observe that we can manipulate the definition of metrics given in [31, obtaining an alternative form, which we call the a priori form, in contrast with the original form of [31, which we call the a posteriori form. Informally, the a posteriori form is the traditional definition, phrased in terms of similarity of probability distributions; the a priori form is instead phrased in terms of expectations. We show that, while on Markov decision processes these two forms coincide, this is not the case for games; moreover, we show that it is the a priori form that provides the canonical metrics for games.

We prove that the a priori metric distance between two states $s$ and $t$ of a concurrent game is equal to $\sup _{\varphi \in \Phi}|\varphi(s)-\varphi(t)|$, where $\Phi$ is the set of properties expressible via the quantitative $\mu$-calculus. This result can be summarized by saying that the quantitative $\mu$-calculus provides a logical characterization for the a priori metrics, similar to the way the ordinary $\mu$-calculus provides a logical characterization of bisimulation. Furthermore, we prove that a priori metrics - and their kernels, the a priori relations - satisfy a reciprocity property, stating that properties expressed in terms of player 1 and player 2 winning conditions have the same distinguishing power. This property is intimately connected to the fact that concurrent games, played with mixed moves, are determined for $\omega$-regular goals [20, 17: the probability that player 1 achieves a goal $\psi$ is one minus the probability that player 2 achieves the goal $\neg \psi$. Reciprocity ensures that there is one, canonical, notion of game equivalence. This is in contrast to the case of alternating bisimulation of [2], in which there are distinct player 1 and player 2 versions, as a consequence of the fact that concurrent games, when played with pure moves, are not determined. The logical characterization and reciprocity result justify our claim that a priori metrics and relations are the canonical notion of metrics, and equivalence, for concurrent games. Neither the logical characterization nor the reciprocity result hold for the a posteriori metrics and relations.

While this introduction focused mostly on metrics and equivalence relations, we also develop results for the asymmetrical versions of these notions, related to simulation. 


\section{Games And Goals}

We will develop metrics for game structures over a set $S$ of states. We start with some preliminary definitions. For a finite set $A$, let $\operatorname{Dist}(A)=\left\{p: A \mapsto[0,1] \mid \sum_{a \in A} p(a)=1\right\}$ denote the set of probability distributions over $A$. We say that $p \in \operatorname{Dist}(A)$ is deterministic if there is $a \in A$ such that $p(a)=1$.

For a set $S$, a valuation over $S$ is a function $f: S \mapsto[0,1]$ associating with every element $s \in S$ a value $0 \leq f(s) \leq 1$; we let $\mathcal{F}$ be the set of all valuations. For $c \in[0,1]$, we denote by $\mathbf{c}$ the constant valuation such that $\mathbf{c}(s)=c$ at all $s \in S$. We order valuations pointwise: for $f, g \in \mathcal{F}$, we write $f \leq g$ iff $f(s) \leq g(s)$ at all $s \in S$; we remark that $\mathcal{F}$, under $\leq$, forms a complete lattice.

Given $a, b \in \mathbb{R}$, we write $a \sqcup b=\max \{a, b\}$, and $a \sqcap b=\min \{a, b\}$; we also let $a \oplus b=\min \{1, \max \{0, a+b\}\}$ and $a \ominus b=\max \{0, \min \{1, a-b\}\}$. We extend $\sqcap, \sqcup,+,-, \oplus, \ominus$ to valuations by interpreting them in pointwise fashion.

A directed metric is a function $d: S^{2} \mapsto \mathbb{R}_{\geq 0}$ which satisfies $d(s, s)=0$ and $d(s, t) \leq$ $d(s, u)+d(u, t)$ for all $s, t, u \in S$. We denote by $\mathcal{M} \subseteq S^{2} \mapsto \mathbb{R}$ the space of all metrics; this space, ordered pointwise, forms a lattice which we indicate with $(\mathcal{M}, \leq)$. Given a metric $d \in \mathcal{M}$, we denote by $\breve{d}$ its opposite version, defined by $\breve{d}(s, t)=d(t, s)$ for all $s, t \in S$; we say that $d$ is symmetrical if $d=\breve{d}$.

2.1. Game Structures. We assume a fixed, finite set $\mathcal{V}$ of observation variables. A (twoplayer, concurrent) game structure $G=\left\langle S,[\cdot]\right.$, Moves $\left., \Gamma_{1}, \Gamma_{2}, \delta\right\rangle$ consists of the following components [1, 5]:

- A finite set $S$ of states.

- A variable interpretation [.] $: \mathcal{V} \times S \mapsto[0,1]$, which associates with each variable $v \in \mathcal{V}$ a valuation $[v]$.

- A finite set Moves of moves.

- Two move assignments $\Gamma_{1}, \Gamma_{2}: S \mapsto 2^{\text {Moves }} \backslash \emptyset$. For $i \in\{1,2\}$, the assignment $\Gamma_{i}$ associates with each state $s \in S$ the nonempty set $\Gamma_{i}(s) \subseteq$ Moves of moves available to player $i$ at state $s$.

- A probabilistic transition function $\delta: S \times$ Moves $\times$ Moves $\mapsto \operatorname{Dist}(S)$, that gives the probability $\delta\left(s, a_{1}, a_{2}\right)(t)$ of a transition from $s$ to $t$ when player 1 plays move $a_{1}$ and player 2 plays move $a_{2}$.

At every state $s \in S$, player 1 chooses a move $a_{1} \in \Gamma_{1}(s)$, and simultaneously and independently player 2 chooses a move $a_{2} \in \Gamma_{2}(s)$. The game then proceeds to the successor state $t \in S$ with probability $\delta\left(s, a_{1}, a_{2}\right)(t)$. We denote by $\operatorname{Dest}\left(s, a_{1}, a_{2}\right)=\{t \in S \mid$ $\left.\delta\left(s, a_{1}, a_{2}\right)(t)>0\right\}$ the set of destination states when actions $a_{1}, a_{2}$ are chosen at $s$. The variables in $\mathcal{V}$ naturally induce an equivalence on states: for states $s, t$, define $s \equiv t$ if for all $v \in \mathcal{V}$ we have $[v](s)=[v](t)$. In the following, unless otherwise noted, the definitions refer to a game structure with components $G=\left\langle S,[\cdot]\right.$, Moves $\left., \Gamma_{1}, \Gamma_{2}, \delta\right\rangle$. For player $i \in\{1,2\}$, we write $\sim i=3-i$ for the opponent. We also consider the following subclasses of game structures.

- Turn-based game structures. A game structure $G$ is turn-based if we can write $S$ as the disjoint union of two sets: the set $S_{1}$ of player 1 states, and the set $S_{2}$ of player 2 states, such that $s \in S_{1}$ implies $\left|\Gamma_{2}(s)\right|=1$, and $s \in S_{2}$ implies $\left|\Gamma_{1}(s)\right|=1$, and further, there 
is a special variable turn $\in \mathcal{V}$, such that $[$ turn $](s)=1$ iff $s \in S_{1}$, and $[$ turn $](s)=0$ iff $s \in S_{2}$ : thus, the variable turn indicates whose turn it is to play at a state.

- Markov decision processes. A game structure $G$ is a Markov decision process (MDP) [8] if only one of the two players has a choice of moves. For $i \in\{1,2\}$, we say that a structure is an $i$-MDP if $\forall s \in S,\left|\Gamma_{\sim i}(s)\right|=1$. For MDPs, we omit the (single) move of the player without a choice of moves, and write $\delta(s, a)$ for the transition function.

- Deterministic game structures. A game structure $G$ is deterministic if, for all $s \in S$, $a_{1} \in$ Moves, and $a_{2} \in$ Moves, there exists a $t \in S$ such that $\delta\left(s, a_{1}, a_{2}\right)(t)=1$; we denote such $t$ by $\tau\left(s, a_{1}, a_{2}\right)$. We sometimes call probabilistic a general game structure, to emphasize the fact that it is not necessarily deterministic.

Note that MDPs can be seen as turn-based games by setting [turn] $=\mathbf{1}$ for 1-MDPs and $[$ turn $]=\mathbf{0}$ for 2-MDPs.

Pure and mixed moves. A mixed move is a probability distribution over the moves available to a player at a state. We denote by $\mathcal{D}_{i}(s)=\operatorname{Dist}\left(\Gamma_{i}(s)\right)$ the set of mixed moves available to player $i \in\{1,2\}$ at $s \in S$. The moves in Moves are called pure moves, in contrast to mixed moves. We extend the transition function to mixed moves. For $s \in S$ and $x_{1} \in \mathcal{D}_{1}(s), x_{2} \in \mathcal{D}_{2}(s)$, we write $\delta\left(s, x_{1}, x_{2}\right)$ for the next-state probability distribution induced by the mixed moves $x_{1}$ and $x_{2}$, defined for all $t \in S$ by

$$
\delta\left(s, x_{1}, x_{2}\right)(t)=\sum_{a_{1} \in \Gamma_{1}(s)} \sum_{a_{2} \in \Gamma_{2}(s)} \delta\left(s, a_{1}, a_{2}\right)(t) x_{1}\left(a_{1}\right) x_{2}\left(a_{2}\right) .
$$

In the following, we sometimes restrict the moves of the players to pure moves. We identify a pure move $a \in \Gamma_{i}(s)$ available to player $i \in\{1,2\}$ at a state $s$ with a deterministic distribution that plays $a$ with probability 1 .

The deterministic setting. The deterministic setting is obtained by considering deterministic game structures, with players restricted to playing pure moves.

2.2. Predecessor operators. Given a valuation $f \in \mathcal{F}$, a state $s \in S$, and two mixed moves $x_{1} \in \mathcal{D}_{1}(s)$ and $x_{2} \in \mathcal{D}_{2}(s)$, we define the expectation of $f$ from $s$ under $x_{1}, x_{2}$ :

$$
\mathbb{E}_{s}^{x_{1}, x_{2}}(f)=\sum_{t \in S} \delta\left(s, x_{1}, x_{2}\right)(t) f(t)
$$

For a game structure $G$, for $i \in\{1,2\}$ we define the valuation transformer $\operatorname{Pre}_{i}: \mathcal{F} \mapsto \mathcal{F}$ by, for all $f \in \mathcal{F}$ and $s \in S$,

$$
\operatorname{Pre}_{i}(f)(s)=\sup _{x_{i} \in \mathcal{D}_{i}(s)} \inf _{x_{\sim i} \in \mathcal{D}_{\sim i}(s)} \mathbb{E}_{s}^{x_{1}, x_{2}}(f) .
$$

Intuitively, $\operatorname{Pre}_{i}(f)(s)$ is the maximal expectation player $i$ can achieve of $f$ after one step from $s$ : this is the classical "one-day" or "next-stage" operator of the theory of repeated games [12]. We also define a deterministic version of this operator, in which players are forced to play pure moves:

$$
\operatorname{Pre}_{i}^{\Gamma}(f)(s)=\max _{x_{i} \in \Gamma_{i}(s)} \min _{x_{\sim i} \in \Gamma_{\sim i}(s)} \mathbb{E}_{s}^{x_{1}, x_{2}}(f) .
$$


2.3. Quantitative $\mu$-calculus. We consider the set of properties expressed by the quantitative $\mu$-calculus $(q \mu)$. As discussed in [16, 7, 21, a large set of properties can be encoded in $q \mu$, spanning from basic properties such as maximal reachability and safety probability, to the maximal probability of satisfying a general $\omega$-regular specification.

Syntax. The syntax of quantitative $\mu$-calculus is defined with respect to the set of observation variables $\mathcal{V}$ as well as a set MVars of calculus variables, which are distinct from the observation variables in $\mathcal{V}$. The syntax is given as follows:

$$
\varphi::=c|v| Z|\neg \varphi| \varphi \vee \varphi|\varphi \wedge \varphi| \varphi \oplus c|\varphi \ominus c| \operatorname{pre}_{1}(\varphi)\left|\operatorname{pre}_{2}(\varphi)\right| \mu Z . \varphi \mid \nu Z . \varphi
$$

for constants $c \in[0,1]$, observation variables $v \in \mathcal{V}$, and calculus variables $Z \in$ MVars. In the formulas $\mu Z . \varphi$ and $\nu Z . \varphi$, we furthermore require that all occurrences of the bound variable $Z$ in $\varphi$ occur in the scope of an even number of occurrences of the complement operator $\neg$. A formula $\varphi$ is closed if every calculus variable $Z$ in $\varphi$ occurs in the scope of a quantifier $\mu Z$ or $\nu Z$. From now on, with abuse of notation, we denote by $q \mu$ the set of closed formulas of $q \mu$. A formula is a player $i$ formula, for $i \in\{1,2\}$, if $\varphi$ does not contain the pre ${ }_{\sim i}$ operator; we denote with $q \mu_{i}$ the syntactic subset of $q \mu$ consisting only of closed player $i$ formulas. A formula is in positive form if the negation appears only in front of observation variables, i.e., in the context $\neg v$; we denote with $q \mu^{+}$and $q \mu_{i}^{+}$the subsets of $q \mu$ and $q \mu_{i}$ consisting only of positive formulas.

We remark that the fixpoint operators $\mu$ and $\nu$ will not be needed to achieve our results on the logical characterization of game relations. They have been included in the calculus because they allow the expression of many interesting properties, such as safety, reachability, and in general, $\omega$-regular properties. The operators $\oplus$ and $\ominus$, on the other hand, are necessary for our results.

Semantics. A variable valuation $\xi: M$ Vars $\mapsto \mathcal{F}$ is a function that maps every variable $Z \in M$ Vars to a valuation in $\mathcal{F}$. We write $\xi[Z \mapsto f]$ for the valuation that agrees with $\xi$ on all variables, except that $Z$ is mapped to $f$. Given a game structure $G$ and a variable valuation $\xi$, every formula $\varphi$ of the quantitative $\mu$-calculus defines a valuation $\llbracket \varphi \rrbracket_{\xi}^{G} \in \mathcal{F}$ (the superscript $G$ is omitted if the game structure is clear from the context):

$$
\begin{aligned}
& \llbracket c \rrbracket_{\xi}=\mathbf{c} \\
& \llbracket v \rrbracket_{\xi}=[v] \\
& \llbracket Z \rrbracket_{\xi}=\xi(Z) \\
& \llbracket \neg \varphi \rrbracket_{\xi}=\mathbf{1}-\llbracket \varphi \rrbracket_{\xi} \\
& \llbracket \varphi\left\{\begin{array}{c}
\oplus \\
\ominus
\end{array}\right\} c \rrbracket_{\xi}=\llbracket \varphi \rrbracket_{\xi}\left\{\begin{array}{l}
\oplus \\
\ominus
\end{array}\right\} \mathbf{c} \\
& \llbracket \varphi_{1}\left\{\begin{array}{c}
\vee \\
\wedge
\end{array}\right\} \varphi_{2} \rrbracket_{\xi}=\llbracket \varphi_{1} \rrbracket_{\xi}\left\{\begin{array}{c}
\sqcup \\
\sqcap
\end{array}\right\} \llbracket \varphi_{2} \rrbracket_{\xi} \\
& \llbracket \operatorname{prre}_{i}(\varphi) \rrbracket_{\xi}=\operatorname{Pre}_{i}\left(\llbracket \varphi \rrbracket_{\xi}\right) \\
& \llbracket\left\{\begin{array}{l}
\mu \\
\nu
\end{array}\right\} Z \cdot \varphi \rrbracket_{\xi}=\left\{\begin{array}{c}
\inf _{\sup } \\
\operatorname{sun}
\end{array}\right\}\left\{f \in \mathcal{F} \mid f=\llbracket \varphi \rrbracket_{\xi[Z \mapsto f]}\right\}
\end{aligned}
$$

where $i \in\{1,2\}$. The existence of the fixpoints is guaranteed by the monotonicity and continuity of all operators and can be computed by Picard iteration [7]. If $\varphi$ is closed, $\llbracket \varphi \rrbracket_{\xi}$ is independent of $\xi$, and we write simply $\llbracket \varphi \rrbracket$.

We also define a deterministic semantics $\llbracket \cdot \rrbracket^{\Gamma}$ for $q \mu$, in which players can select only pure moves in the operators pre $_{1}$, pre $e_{2} \cdot \llbracket \cdot \rrbracket^{\Gamma}$ is defined as $\llbracket \cdot \rrbracket$, except for the clause

$$
\llbracket \operatorname{pre}_{i}(\varphi) \rrbracket_{\xi}^{\Gamma}=\operatorname{Pre}_{i}^{\Gamma}\left(\llbracket \varphi \rrbracket_{\xi}^{\Gamma}\right) .
$$


Example 1. Given a set $T \subseteq S$, the characteristic valuation $\mathbf{T}$ of $T$ is defined by $\mathbf{T}(s)=1$ if $s \in T$, and $\mathbf{T}(s)=0$ otherwise. With this notation, the maximal probability with which player $i \in\{1,2\}$ can ensure eventually reaching $T \subseteq S$ is given by $\llbracket \mu Z$. $\left(\mathbf{T} \vee \operatorname{pre}_{i}(Z)\right) \rrbracket$, and the maximal probability with which player $i$ can guarantee staying in $T$ forever is given by $\llbracket \nu Z .\left(\mathbf{T} \wedge \operatorname{pre}_{i}(Z)\right) \rrbracket($ see, e.g., [7]). The first property is called a reachability property, the second a safety property.

\section{MetriCs}

We are interested in developing a metric on states of a game structure that captures an approximate notion of equivalence: states close in the metric should yield similar values to the players for any winning objective. Specifically, we are interested in defining a bisimulation metric $\left[\simeq_{g}\right] \in \mathcal{M}$ such that for any game structure $G$ and states $s, t$ of $G$, the following continuity property holds:

$$
\left[\simeq_{g}\right](s, t)=\sup _{\varphi \in q \mu}|\llbracket \varphi \rrbracket(s)-\llbracket \varphi \rrbracket(t)| .
$$

In particular, the kernel of the metric, that is, states at distance 0 , are equivalent: each player can get exactly the same value from either state for any objective. Notice that in defining the metric independent of a player, we are expecting our metrics to be reciprocal, that is, invariant under a change of player. Reciprocity is expected to hold since the underlying games we consider are determined - for any game, the value obtained by player 2 is one minus the value obtained by player 1 - and yields canonical metrics on games.

Thus, our metrics will generalize equivalence and refinement relations that have been studied on MDPs and in the deterministic setting. To underline the connection between classical equivalences and the metrics we develop, we write $\left[s \simeq_{g} t\right]$ for $\left[\simeq_{g}\right](s, t)$, so that the desired property of the bisimulation metric can be stated as

$$
\left[s \simeq_{g} t\right]=\sup _{\varphi \in q \mu}|\llbracket \varphi \rrbracket(s)-\llbracket \varphi \rrbracket(t)| .
$$

Metrics of this type have already been developed for Markov decision processes (MDPs) [30, 10. Our construction of metrics for games starts from an analysis of these constructions.

3.1. Metrics for MDPs. We consider the case of 1-MDPs; the case for 2-MDPs is symmetrical. Throughout this subsection, we fix a 1-MDP $\left\langle S\right.$, [.], Moves, $\left.\Gamma_{1}, \Gamma_{2}, \delta\right\rangle$. Before we present the metric correspondent of probabilistic simulation, we first rephrase classical probabilistic (bi)simulation on MDPs [18, 14, 25, 26] as a fixpoint of a relation transformer. As a first step, we lift relations between states to relations between distributions. Given a relation $R \subseteq S \times S$ and two distributions $p, q \in \operatorname{Dist}(S)$, we let $p \sqsubseteq_{R} q$ if there is a function $\Delta: S \times S \rightarrow[0,1]$ such that:

- $\Delta\left(s, s^{\prime}\right)>0$ implies $\left(s, s^{\prime}\right) \in R$;

- $p(s)=\sum_{s^{\prime} \in S} \Delta\left(s, s^{\prime}\right)$ for any $s \in S$;

- $q\left(s^{\prime}\right)=\sum_{s \in S} \Delta\left(s, s^{\prime}\right)$ for any $s^{\prime} \in S$.

To rephrase probabilistic simulation, we define the relation transformer $F: 2^{S \times S} \mapsto 2^{S \times S}$ as follows. For all relations $R \subseteq S \times S$ and $s, t \in S$, we let $(s, t) \in F(R)$ iff

$$
s \equiv t \wedge \forall x_{1} \in \mathcal{D}_{1}(s) . \exists y_{1} \in \mathcal{D}_{1}(t) . \delta\left(s, x_{1}\right) \sqsubseteq_{R} \delta\left(t, y_{1}\right),
$$


for all states $s, t \in S$. Probabilistic simulation is the greatest fixpoint of (3.2); probabilistic bisimulation is the greatest symmetrical fixpoint of (3.2).

To obtain a metric equivalent of probabilistic simulation, we lift the above fixpoint from relations (subsets of $S^{2}$ ) to metrics (maps $S^{2} \mapsto \mathbb{R}$ ). First, we define [三] $\in \mathcal{M}$ for all $s, t \in S$ by $[s \equiv t]=\max _{v \in \mathcal{V}}|[v](s)-[v](t)|$. Second, we lift (3.2) to metrics, defining a metric transformer $H_{\text {post }}^{1 M D P}: \mathcal{M} \mapsto \mathcal{M}$. For all $d \in \mathcal{M}$, let $D\left(\delta\left(s, x_{1}\right), \delta\left(t, y_{1}\right)\right)(d)$ be the distribution distance between $\delta\left(s, x_{1}\right)$ and $\delta\left(t, y_{1}\right)$ with respect to the metric $d$. We will show later how to define such a distribution distance. For $s, t \in S$, we let

$$
H_{\text {post }}^{1 M D P}(d)(s, t)=[s \equiv t] \sqcup \sup _{x_{1} \in \mathcal{D}_{1}(s)} \inf _{y_{1} \in \mathcal{D}_{1}(t)} D\left(\delta\left(s, x_{1}\right), \delta\left(t, y_{1}\right)\right)(d) .
$$

In this definition, the $\forall$ and $\exists$ of (3.2) have been replaced by sup and inf, respectively. Since equivalent states should have distance 0, the simulation metric in MDPs is defined as the least (rather than greatest) fixpoint of (3.3) [30, 10]. Similarly, the bisimulation metric is defined as the least symmetrical fixpoint of (3.3).

For a distance $d \in \mathcal{M}$ and two distributions $p, q \in \operatorname{Dist}(S)$, the distribution distance $D(p, q)(d)$ is a measure of how much "work" we have to do to make $p$ look like $q$, given that moving a unit of probability mass from $s \in S$ to $t \in S$ has cost $d(s, t)$. More precisely, $D(p, q)(d)$ is defined via the trans-shipping problem, as the minimum cost of shipping the distribution $p$ into $q$, with edge costs $d$. Thus, $D(p, q)(d)$ is the solution of the following linear programming (LP) problem over the set of variables $\left\{\lambda_{s, t}\right\}_{s, t \in S}$ :

$$
\begin{gathered}
\text { Minimize } \sum_{s, t \in S} d(s, t) \lambda_{s, t} \\
\text { subject to } \sum_{t \in S} \lambda_{s, t}=p(s), \quad \sum_{s \in S} \lambda_{s, t}=q(t), \quad \lambda_{s, t} \geq 0 .
\end{gathered}
$$

Equivalently, we can define $D(p, q)(d)$ via the dual of the above LP problem [30]. Given a metric $d \in \mathcal{M}$, let $C(d) \subseteq \mathcal{F}$ be the subset of valuations $k \in \mathcal{F}$ such that $k(s)-k(t) \leq d(s, t)$ for all $s, t \in S$. Then the dual formulation is:

$$
\text { Maximize } \sum_{s \in S} p(s) k(s)-\sum_{s \in S} q(s) k(s)
$$

subject to $k \in C(d)$.

The constraint $C(d)$ on the valuation $k$, states that the value of $k$ across states cannot differ by more than $d$. This means, intuitively, that $k$ behaves like the valuation of a $q \mu$ formula: as we will see, the logical characterization implies that $d$ is a bound for the difference in valuation of $q \mu$ formulas across states. Indeed, the logical characterization of the metrics is proved by constructing formulas whose valuation approximate that of the optimal $k$. Plugging (3.4) into (3.3), we obtain:

$$
H_{\text {post }}^{1 M P P}(d)(s, t)=[s \equiv t] \sqcup \sup _{x_{1} \in \mathcal{D}_{1}(s)} \inf _{y_{1} \in \mathcal{D}_{1}(t)} \sup _{k \in C(d)}\left(\mathbb{E}_{s}^{x_{1}}(k)-\mathbb{E}_{t}^{y_{1}}(k)\right) .
$$

We can interpret this definition as follows. State $t$ is trying to simulate state $s$ (this is a definition of a simulation metric). First, state $s$ chooses a mixed move $x_{1}$, attempting to make simulation as hard as possible; then, state $t$ chooses a mixed move $y_{1}$, trying to match the effect of $x_{1}$. Once $x_{1}$ and $y_{1}$ have been chosen, the resulting distance between $s$ and $t$ is equal to the maximal difference in expectation, for moves $x_{1}$ and $y_{1}$, of a valuation 
$k \in C(d)$. We call the metric transformer $H_{\text {post }}^{1 M D P}$ the a posteriori metric transformer: the valuation $k$ in (3.5) is chosen after the moves $x_{1}$ and $y_{1}$ are chosen. We can define an $a$ priori metric transformer, where $k$ is chosen before $x_{1}$ and $y_{1}$ :

$$
H_{\text {prio }}^{1 M D P}(d)(s, t)=[s \equiv t] \sqcup \sup _{k \in C(d)} \sup _{x_{1} \in \mathcal{D}_{1}(s)} \inf _{y_{1} \in \mathcal{D}_{1}(t)}\left(\mathbb{E}_{s}^{x_{1}}(k)-\mathbb{E}_{t}^{y_{1}}(k)\right) .
$$

Intuitively, in the a priori transformer, first a valuation $k \in C(d)$ is chosen. Then, state $t$ must simulate state $s$ with respect to the expectation of $k$. State $s$ chooses a move $x_{1}$, trying to maximize the difference in expectations, and state $t$ chooses a move $y_{1}$, trying to minimize it. The distance between $s$ and $t$ is then equal to the difference in the resulting expectations of $k$.

Theorem 3.1 below states that for MDPs, a priori and a posteriori simulation metrics coincide. In the next section, we will see that this is not the case for games.

Theorem 3.1. For all MDPs, $H_{\text {post }}^{1 M D P}=H_{\text {prio }}^{1 M D P}$.

Proof. Consider two states $s, t \in S$, and a metric $d \in \mathcal{M}$. We have to prove that

$$
\sup _{k} \sup _{x_{1}} \inf _{y_{1}}\left[\mathbb{E}_{s}^{x_{1}}(k)-\mathbb{E}_{t}^{y_{1}}(k)\right]=\sup _{x_{1}} \inf _{y_{1}} \sup _{k}\left[\mathbb{E}_{s}^{x_{1}}(k)-\mathbb{E}_{t}^{y_{1}}(k)\right] .
$$

In the left-hand side, we can exchange the two outer sups. Then, noticing that the difference in expectation is bi-linear in $k$ and $y_{1}$ for a fixed $x_{1}$, that $y_{1}$ is a probability distribution, and that $k$ is chosen from a compact convex subset, we apply the generalized minimax theorem [28] to exchange $\sup _{k} \inf _{y_{1}}$ into $\inf _{y_{1}} \sup _{k}$, thus obtaining the right-hand side.

The metrics defined above are logically characterized by $q \mu$. Precisely, let $[\sim] \in \mathcal{M}$ be the least symmetrical fixpoint of $H_{\text {prio }}^{1 M D P}=H_{\text {post }}^{1 M P P}$. Then, Lemma 5.24 and Corollary 5.25 of [10], (originally stated for $H_{\text {post }}^{1 M D P}$ ) state that for all states $s, t$ of a 1-MDP, we have

$$
[s \sim t]=\sup _{\varphi \in q \mu}|\llbracket \varphi \rrbracket(s)-\llbracket \varphi \rrbracket(t)| .
$$

3.2. Metrics for Concurrent Games. We now extend the simulation and bisimulation metrics from MDPs to general game structures. As we shall see, unlike for MDPs, the a priori and the a posteriori metrics do not coincide over games. In particular, we show that the a priori formulation satisfies both a tight logical characterization as well as reciprocity while, perhaps surprisingly, the more natural a posteriori version does not.

A posteriori metrics are defined via the metric transformer $H_{\sqsubseteq_{1}}: \mathcal{M} \mapsto \mathcal{M}$ as follows, for all $d \in \mathcal{M}$ and $s, t \in S$ :

$$
\begin{aligned}
H_{\sqsubseteq_{1}}(d)(s, t) & =[s \equiv t] \sqcup \sup _{x_{1} \in \mathcal{D}_{1}(s)} \inf _{y_{1} \in \mathcal{D}_{1}(t)} \sup _{y_{2} \in \mathcal{D}_{2}(t)} \inf _{x_{2} \in \mathcal{D}_{2}(s)} D\left(\delta\left(s, x_{1}, x_{2}\right), \delta\left(t, y_{1}, y_{2}\right), d\right) \\
& =[s \equiv t] \sqcup \sup _{x_{1} \in \mathcal{D}_{1}(s)} \inf _{y_{1} \in \mathcal{D}_{1}(t)} \sup _{y_{2} \in \mathcal{D}_{2}(t)} \inf _{x_{2} \in \mathcal{D}_{2}(s)} \sup _{k \in C(d)}\left(\mathbb{E}_{s}^{x_{1}, x_{2}}(k)-\mathbb{E}_{t}^{y_{1}, y_{2}}(k)\right) .
\end{aligned}
$$


A priori metrics are defined by bringing the $\sup _{k}$ outside. Precisely, we define a metric transformer $H_{\preceq_{1}}: \mathcal{M} \mapsto \mathcal{M}$ as follows, for all $d \in \mathcal{M}$ and $s, t \in S$ :

$$
\begin{aligned}
H_{\preceq_{1}}(d)(s, t) & =[s \equiv t] \sqcup \sup _{k \in C(d)} \sup _{x_{1} \in \mathcal{D}_{1}(s)} \inf _{y_{1} \in \mathcal{D}_{1}(t)} \sup _{y_{2} \in \mathcal{D}_{2}(t)} \inf _{x_{2} \in \mathcal{D}_{2}(s)}\left(\mathbb{E}_{s}^{x_{1}, x_{2}}(k)-\mathbb{E}_{t}^{y_{1}, y_{2}}(k)\right) \\
& =[s \equiv t] \sqcup \sup _{k \in C(d)}\left[\sup _{x_{1} \in \mathcal{D}_{1}(s)} \inf _{x_{2} \in \mathcal{D}_{2}(s)} \mathbb{E}_{s}^{x_{1}, x_{2}}(k)-\sup _{y_{1} \in \mathcal{D}_{1}(t)} \inf _{y_{2} \in \mathcal{D}_{2}(t)} \mathbb{E}_{t}^{y_{1}, y_{2}}(k)\right] \\
& =[s \equiv t] \sqcup \sup _{k \in C(d)}\left(\operatorname{Pre}_{1}(k)(s)-\operatorname{Pre}_{1}(k)(t)\right) .
\end{aligned}
$$

First, we show that $H_{\preceq_{1}}$ and $H_{\coprod_{1}}$ are monotonic in the lattice of metrics $(\mathcal{M}, \leq)$.

Lemma 3.2. The functions $H_{\preceq_{1}}$ and $H_{\bigsqcup_{1}}$ are monotonic in the lattice of metrics $(\mathcal{M}, \leq)$.

Proof. For $d, d^{\prime} \in \mathcal{M}, d \leq d^{\prime}$ implies $C(d) \subseteq C\left(d^{\prime}\right)$, and hence $\sup _{k \in C(d)}\left(\operatorname{Pre}_{1}(k)(s)-\right.$ $\left.\operatorname{Pre}_{1}(k)(t)\right) \leq \sup _{k \in C\left(d^{\prime}\right)}\left(\operatorname{Pre}_{1}(k)(s)-\operatorname{Pre}_{1}(k)(t)\right)$. This shows the monotonicity of $H_{\preceq_{1}}$.

The monotonicity of $H_{\sqsubseteq_{1}}$ can be shown in a similar fashion. From $d \leq d^{\prime}$, reasoning as before we obtain

$$
\sup _{k \in C(d)}\left(\mathbb{E}_{s}^{x_{1}, x_{2}}(k)-\mathbb{E}_{t}^{y_{1}, y_{2}}(k)\right) \leq \sup _{k \in C\left(d^{\prime}\right)}\left(\mathbb{E}_{s}^{x_{1}, x_{2}}(k)-\mathbb{E}_{t}^{y_{1}, y_{2}}(k)\right) .
$$

The result then follows from the monotonicity of the operators $\sup _{x_{1} \in \mathcal{D}_{1}(s)}, \inf _{y_{1} \in \mathcal{D}_{1}(t)}$, $\sup _{y_{2} \in \mathcal{D}_{2}(t)}, \inf _{x_{2} \in \mathcal{D}_{2}(s)}$.

On the basis of this lemma, we can define the least fixpoints of $H_{\unlhd_{1}}$ and $H_{\varliminf_{1}}$, which will yield our game simulation and bisimulation metrics.

Definition 3.3. A priori metrics:

- The a priori simulation metric $\left[\preceq_{1}\right]$ is the least fixpoint of $H_{\preceq_{1}}$.

- The a priori bisimulation metric $\left[\simeq_{1}\right]$ is the least symmetrical fixpoint of $H_{{ }_{1}}$.

A posteriori metrics:

- The a posteriori game simulation metric [ $\left.\sqsubseteq_{1}\right]$ is the least fixpoint of $H_{\bigsqcup_{1}}$.

- The a posteriori game bisimulation metric $\left[\cong_{1}\right]$ is the least symmetrical fixpoint of $H_{\sqsubseteq_{1}}$. By exchanging the roles of the players, we define the metric transformers $H_{\unlhd_{2}}$ and $H_{\bigsqcup_{2}}$, and the metrics $\left[\preceq_{2}\right],\left[\simeq_{2}\right],\left[\complement_{2}\right],\left[\varrho_{2}\right]$.

We note that the a posteriori simulation metric $\left[\varrho_{1}\right]$ has been introduced in [6, 19]. We also note that the a posteriori bisimulation metric $\left[\cong_{i}\right]$ can be defined as the least fixpoint of $H_{\cong_{i}}: \mathcal{M} \mapsto \mathcal{M}$, defined for all $d \in \mathcal{M}$ and $i \in\{1,2\}$ by

$$
H \cong_{1}(d)=H_{\sqsubseteq_{1}}(d) \sqcup O p p\left(H_{\sqsubseteq_{1}}(d)\right),
$$

where $O p p(d)=\breve{d}$ denotes the opposite of a metric $d$. Similarly, the a priori bisimulation metric $\left[\simeq_{i}\right]$ can be defined as the least fixpoint of $H_{\simeq_{i}}: \mathcal{M} \mapsto \mathcal{M}$, defined for all $d \in \mathcal{M}$ and $i \in\{1,2\}$ by

$$
H_{\simeq_{1}}(d)=H_{\preceq_{1}}(d) \sqcup O p p\left(H_{\preceq_{1}}(d)\right) .
$$

We wish to show that the metrics of Definition 3.3 can be computed via Picard iteration. To this end, it is necessary to show that the operators $H_{\varliminf_{1}}$ and $H_{\preceq_{1}}$ on the lattice $(\mathcal{M}, \leq)$ are upper semi-continuous. In fact, a very similar proof shows that the operators are lower semi-continuous, and thus, continuous; we omit the proof of this more general fact as it is not required for the desired result about the applicability of Picard iteration. 
Lemma 3.4. The operators $H_{\preceq_{1}}$ and $H_{\bigsqcup_{1}}$ on the lattice $(\mathcal{M}, \leq)$ are upper semi-continuous.

Proof. Let $D \subseteq \mathcal{M}$ be an arbitrary set of distances, and let $d^{*}=\sup D$; note that $d^{*}$ exists, as $(\mathcal{M}, \leq)$ is a complete lattice.

We first prove the result for $H_{\preceq_{1}}$. We need to prove that $H_{\preceq_{1}}(\sup D)=\sup _{d \in D} H_{\unlhd_{1}}(d)$, which we abbreviate $H_{\preceq_{1}}(\sup D)=\sup H_{\preceq_{1}}(D)$. In one direction, $H_{\preceq_{1}}(\sup D) \geq \sup H_{\preceq_{1}}(D)$ follows from the monotonicity of $H_{\preceq_{1}}$ (Lemma 3.2). In the other direction, we will show that for all $\epsilon>0$, there is $d \in D$ such that $\left|H_{\unlhd_{1}}\left(d^{*}\right)-H_{\preceq_{1}}(d)\right| \leq \epsilon$, where for $d, d^{\prime} \in \mathcal{M}$, $\left|d-d^{\prime}\right|$ is the 1-norm distance between $d$ and $d^{\prime}$. For convenience, let $G(k) \in \mathcal{M}$ be defined as $G(k)(s, t)=\operatorname{Pre}_{1}(k)(s)-\operatorname{Pre}_{1}(k)(t)$, so that we can write $H_{\preceq_{1}}(d)=[s \equiv t] \sqcup \sup _{k \in C(d)} G(k)$.

Given $\epsilon>0$, choose $d \in D$ such that for all $s, t \in S$, we have $d(s, t) / d^{*}(s, t) \geq 1-\epsilon / 4$ if $d^{*}(s, t)>0$, and $d(s, t)=0$ if $d^{*}(s, t)=0$. Note that for all $k \in C\left(d^{*}\right)$, we have $(1-\epsilon / 4) k \in C(d)$ and $|k-(1-\epsilon / 4) k| \leq \epsilon / 4$, as $|k| \leq 1$. Thus, $d \in D$ is such that for all $k \in C\left(d^{*}\right)$, there is $k^{\prime} \in C(d)$ with $\left|k-k^{\prime}\right| \leq \epsilon / 4$. In other words, $d$ is such that the Hausdorff distance between $C\left(d^{*}\right)$ and $C(d)$ is at most $\epsilon / 4$. We now prove that for this $d$, we have

$$
\left|\sup _{k \in C\left(d^{*}\right)} G(k)-\sup _{k \in C(d)} G(k)\right| \leq \epsilon .
$$

In fact, let $k^{*} \in C\left(d^{*}\right)$ be such that

$$
\left|G\left(k^{*}\right)-\sup _{k \in C\left(d^{*}\right)} G(k)\right| \leq \epsilon / 2 .
$$

and let $k^{\prime} \in C(d)$ be such that $\left|k^{*}-k^{\prime}\right| \leq \epsilon / 4$. For $s, t \in S$, we have by definition $G\left(k^{*}\right)(s, t)=\operatorname{Pre}_{1}\left(k^{*}\right)(s)-\operatorname{Pre}_{1}\left(k^{*}\right)(t)$; let

$$
x_{1}(s)=\arg \sup _{x \in \mathcal{D}_{1}(s)} \inf _{y \in \mathcal{D}_{2}(s)} \mathbb{E}_{s}^{x, y}\left(k^{*}\right) .
$$

By employing $x_{1}(s)$ at all $s \in S$, player 1 can guarantee

$$
\left|G\left(k^{\prime}\right)(s, t)-G\left(k^{*}\right)(s, t)\right| \leq \epsilon / 2,
$$

which together with (3.13) leads to (3.12). In turn, (3.12) yields the result.

We can prove the result for $H_{\bigsqcup_{1}}$ following a similar argument. Precisely, in one direction, $H_{\sqsubseteq_{1}}(\sup D) \geq \sup H_{\sqsubseteq_{1}}(D)$ follows from the monotonicity of $H_{\sqsubseteq_{1}}$ (Lemma 3.2). In the other direction, we will show that for all $\epsilon>0$, there is $d \in D$ such that $\left|H_{\sqsubseteq_{1}}\left(d^{*}\right)-H_{\sqsubseteq_{1}}(d)\right| \leq \epsilon$, where for $d, d^{\prime} \in \mathcal{M},\left|d-d^{\prime}\right|$ is the 1-norm distance between $d$ and $d^{\prime}$. Again, let $d$ be such that the Hausdorff distance between $C\left(d^{*}\right)$ and $C(d)$ is at most $\epsilon / 2$. For such a $d$, we have that for all $s, t \in S$, and $x_{1} \in \mathcal{D}_{1}(s), y_{1} \in \mathcal{D}_{1}(t), x_{2} \in \mathcal{D}_{2}(s), y_{2} \in \mathcal{D}_{2}(t)$,

$$
\left|\sup _{k \in C\left(d^{*}\right)}\left(\mathbb{E}_{s}^{x_{1}, x_{2}}(k)-\mathbb{E}_{t}^{y_{1}, y_{2}}(k)\right)-\sup _{k \in C(d)}\left(\mathbb{E}_{s}^{x_{1}, x_{2}}(k)-\mathbb{E}_{t}^{y_{1}, y_{2}}(k)\right)\right| \leq \epsilon,
$$

and this leads easily to the result.

This result implies that we can compute $\left[\preceq_{1}\right]$ as the fixpoint of $H_{\preceq_{1}}$ via Picard iteration; we denote by $d_{n}=H_{\swarrow_{1}}^{n}(\mathbf{0})$ the $n$-iterate of this. Similarly, we can compute [ $\left.\sqsubseteq_{1}\right]$ as the fixpoint of $H_{\sqsubseteq_{1}}$ via Picard iteration.

Theorem 3.5. The following assertions hold, for $i \in\{1,2\}$ :

(1) Let $d_{0}=d_{0}^{\prime}=\mathbf{0}$, and for $n \geq 0$, let

$$
d_{n+1}=H_{\preceq_{i}}\left(d_{n}\right) \quad \text { and } \quad d_{n+1}^{\prime}=H_{\sqsubseteq_{i}}\left(d_{n}^{\prime}\right) .
$$

We have $\lim _{n \rightarrow \infty} d_{n}=\left[\preceq_{i}\right]$ and $\lim _{n \rightarrow \infty} d_{n}^{\prime}=\left[\sqsubseteq_{i}\right]$. 
(2) Let $b_{0}=b_{0}^{\prime}=\mathbf{0}$, and for $n \geq 0$, let

$$
b_{n+1}=H_{\preceq_{i}}\left(b_{n}\right) \sqcup O p p\left(H_{\unlhd_{i}}\left(b_{n}\right)\right) \text { and } b_{n+1}^{\prime}=H_{\varliminf_{i}}\left(b_{n}^{\prime}\right) \sqcup O p p\left(H_{\sqsubseteq_{i}}\left(b_{n}^{\prime}\right)\right) \text {. }
$$

We have $\lim _{n \rightarrow \infty} b_{n}=\left[\simeq_{i}\right]$ and $\lim _{n \rightarrow \infty} b_{n}^{\prime}=\left[\cong_{i}\right]$.

Proof. The statements follow from the definitions of the metrics, and from Lemmas 3.2 and 3.4 .

We now show some basic properties of these metrics. First, we show that the a priori fixpoints give a (directed) metric, i.e., they are non-negative and satisfy the triangle inequality. We also prove that the a priori and a posteriori metrics are distinct. We then focus on the a priori metrics, and show, through our results, that they are the natural metrics for concurrent games.

Theorem 3.6. For all game structures $G$, and all states $s, t, u$ of $G$, we have,

(1) $\left[s \preceq_{1} t\right] \geq 0$ and $\left[s \preceq_{1} u\right] \leq\left[s \preceq_{1} t\right]+\left[t \preceq_{1} u\right]$.

(2) $\left[s \sqsubseteq_{1} t\right] \geq 0$ and $\left[s \sqsubseteq_{1} u\right] \leq\left[s \sqsubseteq_{1} t\right]+\left[t \sqsubseteq_{1} u\right]$.

Proof. We prove the following statement: if $d \in \mathcal{M}$ is a directed metric, then:

(1) $H_{\preceq_{1}}(d)$ is a directed metric;

(2) $H_{\sqsubseteq_{1}}(d)$ is a directed metric.

The theorem then follows by induction on the Picard iteration with which the a priori and a posteriori metrics can be computed (Theorem [3.5). We prove the result first for the a priori metric.

First, from $d^{\prime}=H_{\preceq_{1}}(d)$ and $[\equiv] \geq 0$, we immediately have $d^{\prime} \geq 0$ (where inequalities are interpreted in pointwise fashion).

To prove the triangle inequality, we observe that $[s \equiv t]+[t \equiv u] \geq[s \equiv u]$ for all $s, t, u \in S$. Also,

$$
\begin{array}{r}
\sup _{k \in C(d)}\left(\operatorname{Pre}_{1}(k)(s)-\operatorname{Pre}_{1}(k)(t)\right)+\sup _{k \in C(d)}\left(\operatorname{Pre}_{1}(k)(t)-\operatorname{Pre}_{1}(k)(u)\right) \\
\geq \sup _{k \in C(d)}\left(\operatorname{Pre}_{1}(k)(s)-\operatorname{Pre}_{1}(k)(t)+\operatorname{Pre}_{1}(k)(t)-\operatorname{Pre}_{1}(k)(u)\right) \\
=\sup _{k \in C(d)}\left(\operatorname{Pre}_{1}(k)(s)-\operatorname{Pre}_{1}(k)(u)\right) .
\end{array}
$$

Thus, we obtain

$$
\begin{aligned}
& \quad H_{\preceq_{1}}(d)(s, t)+H_{\preceq_{1}}(d)(t, u) \\
& =\left([s \equiv t] \sqcup \sup _{k \in C(d)}\left(\operatorname{Pre}_{1}(k)(s)-\operatorname{Pre}_{1}(k)(t)\right)\right)+\left([t \equiv u] \sqcup \sup _{k \in C(d)}\left(\operatorname{Pre}_{1}(k)(t)-\operatorname{Pre}_{1}(k)(u)\right)\right) \\
& \quad \geq\left([s \equiv u] \sqcup \sup _{k \in C(d)}\left(\operatorname{Pre}_{1}(k)(s)-\operatorname{Pre}_{1}(k)(u)\right)\right)=H_{\preceq_{1}}(d)(s, u),
\end{aligned}
$$

leading to the result.

For the a posteriori metric, let $d^{\prime}=H_{\sqsubseteq_{1}}(d)$; again, we can prove $d^{\prime} \geq 0$ as in the a priori case. To prove the triangle inequality for $d^{\prime}$, for $s, t \in S$, and for distributions $x_{1} \in \mathcal{D}_{1}(s)$ and $y_{1} \in \mathcal{D}_{1}(t)$, it is convenient to let

$$
G\left(x_{1}, y_{1}\right)(s, t)=\sup _{y_{2} \in \mathcal{D}_{2}(t)} \inf _{x_{2} \in \mathcal{D}_{2}(s)} \sup _{k \in C(d)}\left(\mathbb{E}_{s}^{x_{1}, x_{2}}(k)-\mathbb{E}_{t}^{y_{1}, y_{2}}(k)\right),
$$


With this notation, for $s, t, u \in S$, we have

$$
H_{\sqsubseteq_{1}}(d)(s, u)=[s \equiv u] \sqcup \sup _{x_{1} \in \mathcal{D}_{1}(s)} \inf _{z_{1} \in \mathcal{D}_{1}(u)} G\left(x_{1}, z_{1}\right)(s, u) .
$$

Intuitively, the quantity $G\left(x_{1}, z_{1}\right)(s, u)$ is the distance between $s$ and $u$ computed in the 2-MDP obtained when player 1 plays $x_{1}$ at $s$ and $z_{1}$ at $u$. As a consequence of Theorem 3.1 (interpreted over 2-MDPs), and of the previous proof for the a-priori case, we have that

$$
G\left(x_{1}, z_{1}\right)(s, u) \leq G\left(x_{1}, y_{1}\right)(s, t)+G\left(y_{1}, z_{1}\right)(t, u) .
$$

for all $x_{1} \in \mathcal{D}_{1}(s), y_{1} \in \mathcal{D}_{1}(t)$, and $z_{1} \in \mathcal{D}_{1}(u)$. This observation will be useful in the following.

For any $\epsilon>0$, let $x_{1}^{*}$ realize the sup in (3.16) within $\epsilon$, that is,

$$
\inf _{z_{1} \in \mathcal{D}_{1}(u)} G\left(x_{1}^{*}, z_{1}\right)(s, u) \geq \sup _{x_{1} \in \mathcal{D}_{1}(s)} \inf _{z_{1} \in \mathcal{D}_{1}(u)} G\left(x_{1}, z_{1}\right)(s, u)-\epsilon,
$$

and let $z_{1}^{*}$ realize the inf of the left-hand side of (3.18) also within $\epsilon$. Intuitively, $x_{1}^{*}$ is the player-1 distribution at $s$ that is hardest to imitate from $u$, and $z_{1}^{*}$ is the best imitation of $x_{1}^{*}$ available at $u$. In the same fashion, let $y_{1}^{*}$ realize the inf within $\epsilon \operatorname{in} \inf _{y_{1} \in \mathcal{D}_{1}(t)} G\left(x_{1}^{*}, y_{1}\right)(s, t)$, and let $z_{1}^{\prime}$ realize the inf within $\epsilon$ in $\inf _{z_{1} \in \mathcal{D}_{1}(u)} G\left(y_{1}^{*}, z_{1}\right)(t, u)$. In intuitive terms, $y_{1}^{*}$ is the imitator of $x_{1}^{*}$ in $t$, and $z_{1}^{\prime}$ is the imitator of $y_{1}^{*}$ in $u$.

We consider two cases. If $[s \equiv u]=1$, then we are sure that the triangle inequality

$$
d^{\prime}(s, u) \leq d^{\prime}(s, t)+d^{\prime}(t, u),
$$

holds. Otherwise, note that

$$
d^{\prime}(s, u) \leq G\left(x_{1}^{*}, z_{1}^{*}\right)(s, u)+2 \epsilon .
$$

Since $x_{1}^{*}$ is not necessarily the distribution at $s$ that is hardest to imitate from $t$, and since $y_{1}^{*}$ is not necessarily the distribution at $t$ that is hardest to imitate from $u$, we also have:

$$
d^{\prime}(s, t) \geq G\left(x_{1}^{*}, y_{1}^{*}\right)(s, t)-\epsilon \quad d^{\prime}(t, u) \geq G\left(y_{1}^{*}, z_{1}^{\prime}\right)(t, u)-\epsilon .
$$

Since the triangle inequality holds for MDPs, as stated by (3.17), we have

$$
G\left(x_{1}^{*}, z_{1}^{\prime}\right)(s, u) \leq G\left(x_{1}^{*}, y_{1}^{*}\right)(s, t)+G\left(y_{1}^{*}, z_{1}^{\prime}\right)(t, u) \leq d^{\prime}(s, t)+d^{\prime}(t, u)+2 \epsilon .
$$

Since $z_{1}^{*}$ is the best imitator of $x_{1}^{*}$ at $u$, we also have

$$
G\left(x_{1}^{*}, z_{1}^{*}\right)(s, u)-\epsilon \leq G\left(x_{1}^{*}, z_{1}^{\prime}\right)(s, u),
$$

which together with (3.22) yields

$$
G\left(x_{1}^{*}, z_{1}^{*}\right)(s, u) \leq d^{\prime}(s, t)+d^{\prime}(t, u)+3 \epsilon .
$$

From the choice of $x_{1}^{*}$, this finally leads to

$$
d^{\prime}(s, u) \leq d^{\prime}(s, t)+d^{\prime}(t, u)+5 \epsilon,
$$

for all $\epsilon>0$, which yields the desired triangle inequality (3.19). 


\begin{tabular}{|r||c|c||c|c|}
\hline$\delta(t, *, *)(w)$ & $f$ & $g$ \\
\hline \hline$b$ & $1 / 9$ & $5 / 9$ \\
$c$ & $4 / 9$ & $8 / 9$ \\
\hline \multicolumn{6}{|c||}{$b(t, *, *)(u)$} & $f$ & $g$ \\
\hline \hline$(s, *, *)(w)$ & $f$ & $g$ \\
$c$ & $5 / 9$ & $4 / 9$ \\
\hline \hline$a$ & $1 / 3$ & $2 / 3$ \\
\hline \hline$\delta(s, *, *)(u)$ & $f$ & $g$ \\
\hline$a$ & $2 / 3$ & $1 / 3$ \\
\hline
\end{tabular}

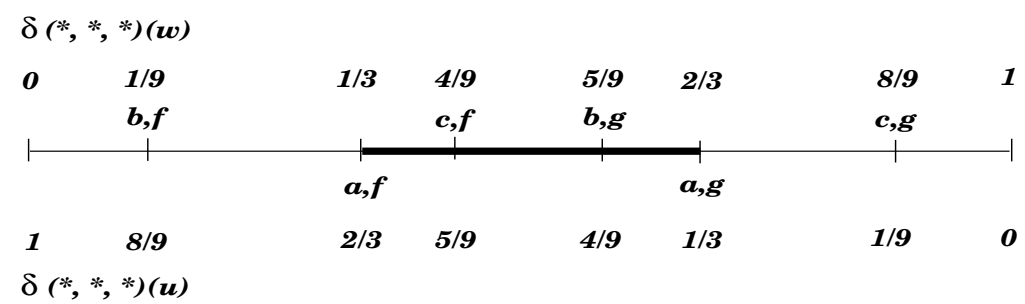

Figure 1: A game that shows that the a priori and the a posteriori metrics may not coincide. The tables above show the transition probabilities from states $t$ and $s$ to states $w$ and $u$ for pure moves of the two players. The row player is player 1 and the column player is player 2 . The line below is the two dimensional probability simplex that shows the transition probabilities induced by convex combinations of pure moves of the two players.

3.3. A priori and a posteriori metrics are distinct. First, we show that a priori and a posteriori metrics are distinct in general: the a priori metric never exceeds the a posteriori one, and there are concurrent games where it is strictly smaller. Intuitively, this can be explained as follows. Simulation entails trying to simulate the expectation of a valuation $k$, as we see from (3.8), (3.9). It is easier to simulate a state $s$ from a state $t$ if the valuation is known in advance, as in a priori metrics (3.9), than if the valuation $k$ is chosen after all the moves have been chosen, as in a posteriori metrics (3.8).

As a special case, we shall see that equality holds for turn-based game structures, in addition to MDPs as we have seen in the previous subsection.

Theorem 3.7. The following assertions hold.

(1) For all game structures $G$, and for all states $s, t$ of $G$, we have $\left[s \preceq_{1} t\right] \leq\left[s \sqsubseteq_{1} t\right]$.

(2) There is a game structure $G$, and states $s, t$ of $G$, such that $\left[s \preceq_{1} t\right]=0$ and $\left[s \sqsubseteq_{1} t\right]>0$.

(3) For all turn-based game structures, we have $\left[\preceq_{1}\right]=\left[\sqsubseteq_{1}\right]$.

Proof. The first assertion is a consequence of the fact that, for all functions $f: \mathbb{R}^{2} \mapsto \mathbb{R}$, we have $\sup _{x} \inf _{y} f(x, y) \leq \inf _{y} \sup _{x} f(x, y)$. By repeated applications of this, we can show that, for all $d \in \mathcal{M}$, we have $H_{\preceq}(d) \leq H_{\sqsubseteq}(d)$ (with pointwise ordering). The result then follows from the monotonicity of $H_{\preceq}$ and $\bar{H}_{\sqsubseteq}$.

For the second assertion, we give an example where a priori distances are strictly less than a posteriori distances. Consider a game with states $S=\{s, t, u, w\}$. States $u$ and $w$ are sink states with $[u \equiv w]=1$; states $s$ and $t$ are such that $[s \equiv t]=0$. At states $s$ and $t$, player 2 has moves $\{f, g\}$. Player- 1 has a single move $\{a\}$ at state $s$, and moves $\{b, c\}$ at state $t$. The moves from $s$ and $t$ lead to $u$ and $w$ with transition probabilities indicated in 
Figure 1. In the figure, the point $b, f$ indicates the probability of going to $u$ and $w$ when the move pair $(b, f)$ is played, with $\delta(s, b, f)(u)+\delta(s, b, f)(w)=1$; similarly for the other move pairs. The thick line segment between the points $a, f$ and $a, g$ represents the transition probabilities arising when player 1 plays move $a$, and player 2 plays a mixed move (a mix of $f$ and $g$ ).

We show that, in this game, we have $\left[\begin{array}{lll}s & \sqsubseteq_{1} & t\end{array}\right]>0$. Consider the metric $d$ where $d(u, w)=1$ (recall that $[u \equiv w]=1$, and note the other distances do not matter, since $u$, $w$ are the only two destinations). We need to show

$$
\forall y_{1} \in \mathcal{D}_{1}(t) . \exists y_{2} \in \mathcal{D}_{2}(t) . \forall x_{2} \in \mathcal{D}_{2}(s) . \exists k \in C(d) .\left(\mathbb{E}_{s}^{a, x_{2}}(k)-\mathbb{E}_{t}^{y_{1}, y_{2}}(k)\right)>0 .
$$

Consider any mixed move $y_{1}=\alpha b+(1-\alpha) c$, where $b, c$ are the moves available to player 1 at $t$, and $0 \leq \alpha \leq 1$. If $\alpha \geq \frac{1}{2}$, choose move $f$ from $t$ as $y_{2}$, and choose $k(w)=1, k(u)=0$. Otherwise, choose move $g$ from $t$ as $y_{2}$, and choose $k(w)=0, k(u)=1$. With these choices, the transition probability $\delta\left(t, y_{1}, y_{2}\right)$ will fall outside of the segment $[(a, f),(a, g)]$ in Figure 1. Thus, with the choice of $k$ above, we ensure that the difference in (3.25) is always positive.

To show that in the game we have $\left[s \preceq_{1} t\right]=0$, it suffices to show (given that $\left[s \preceq_{1}\right.$ $t] \geq 0)$ that

$$
\forall k \in C(d) . \exists y_{1} \in \mathcal{D}_{1}(t) . \forall y_{2} \in \mathcal{D}_{2}(t) . \exists x_{2} \in \mathcal{D}_{2}(s) .\left(\mathbb{E}_{s}^{a, x_{2}}(k)-\mathbb{E}_{t}^{y_{1}, y_{2}}(k)\right) \leq 0 .
$$

If $k(u)=k(w)$, the result is immediate. Assume otherwise, that $k(u)<k(w)$, and choose $y_{1}=c$. For every $y_{2}$, the distribution over successor states (and of $k$-expectations) will be in the interval $[(c, f),(c, g)]$ in Figure 1. By choosing $x_{2}=f$, we have that $\mathbb{E}_{s}^{a, f}(k)<\mathbb{E}_{t}^{c, y_{2}}(k)$ for all $y_{2} \in \mathcal{D}_{2}(t)$, leading to the result. Similarly if $k(u)>k(w)$, by choosing $y_{1}=b$, the distribution over successor states (and of $k$-expectations) will now be in the interval $[(b, f),(b, g)]$. By choosing $x_{2}=g$, we have that $\mathbb{E}_{s}^{a, g}(k)<\mathbb{E}_{t}^{b, y_{2}}(k)$ for all $y_{2} \in \mathcal{D}_{2}(t)$, again leading to the result.

The last assertion of the theorem is proved in the same way as Theorem 3.1.

3.4. Reciprocity of $\boldsymbol{a}$ priori metric. The previous theorem establishes that the a priori and a posteriori metrics are in general distinct. We now prove that it is the a priori metric, rather than the a posteriori one, that enjoys reciprocity, and that provides a (quantitative) logical characterization of $q \mu$. We begin by considering reciprocity.

Theorem 3.8. The following assertions hold.

(1) For all game structures $G$, we have $\left[\preceq_{1}\right]=\left[\succeq_{2}\right]$, and $\left[\simeq_{1}\right]=\left[\simeq_{2}\right]$.

(2) There is a concurrent game structure $G$, with states $s$ and $t$, where $\left[\Xi_{1}\right] \neq\left[\Xi_{2}\right]$.

(3) There is a concurrent game structure $G$, with states $s$ and $t$, where $\left[\cong_{1}\right] \neq\left[\cong_{2}\right]$.

Proof. For the first assertion, it suffices to show that, for all $d \in \mathcal{M}$, and states $s, t \in S$, we have $H_{\preceq_{1}}(d)(s, t)=H_{\preceq_{2}}(\breve{d})(t, s)$. We proceed as follows:

$$
\begin{aligned}
& \sup _{k \in C(d)}\left(\operatorname{Pre}_{1}(k)(s)-\operatorname{Pre}_{1}(k)(t)\right) \\
= & \sup _{k \in C(d)}\left(-\operatorname{Pre}_{2}(1-k)(s)+\operatorname{Pre}_{2}(1-k)(t)\right) \\
= & \sup _{k \in C(\breve{d})}\left(\operatorname{Pre}_{2}(k)(t)-\operatorname{Pre}_{2}(k)(s)\right) .
\end{aligned}
$$


The step from (3.26) to (3.27) uses $\operatorname{Pre}_{1}(k)(s)=1-\operatorname{Pre}_{2}(1-k)(s)$ [32, 7], and the step from (3.27) to (3.28) uses the change of variables $k \rightarrow 1-k$.

For the second assertion, consider again the game of Figure 1, We will show that $\left[t \sqsubseteq_{2} s\right]=0$. Together with $\left[s \sqsubseteq_{1} t\right]>0$, as shown in the proof of Theorem [3.7, this leads to the result. To obtain the result, we will prove that for all $d$, we have:

$$
\forall y_{2} \in \mathcal{D}_{2}(t) . \exists x_{2} \in \mathcal{D}_{2}(s) . \exists y_{1} \in \mathcal{D}_{1}(t) . \forall k \in C(d) .\left(\mathbb{E}_{t}^{y_{2}, y_{1}}(k)-\mathbb{E}_{s}^{x_{2}, a}(k)\right)=0 .
$$

where we have used the fact that player 1 at $s$ plays $x_{1}=a$. Any mixed move $y_{2} \in \mathcal{D}_{2}(t)$ can be written as $y_{2}=\alpha f+(1-\alpha) g$ for $0 \leq \alpha \leq 1$. Choose $y_{1}=\alpha c+(1-\alpha) b$, and

$$
x_{2}=\alpha\left(\frac{2}{3} f+\frac{1}{3} g\right)+(1-\alpha)\left(\frac{1}{3} f+\frac{2}{3} g\right) .
$$

Under this choice of mixed moves, we have:

$$
\begin{aligned}
& \delta\left(t, y_{1}, y_{2}\right)(w)=\frac{4}{9} \alpha^{2}+\alpha(1-\alpha)+\frac{5}{9}(1-\alpha)^{2}=\frac{5}{9}-\frac{1}{9} \alpha \\
& \delta\left(s, x_{1}, x_{2}\right)(w)=\alpha\left(\frac{2}{3} \cdot \frac{1}{3}+\frac{1}{3} \cdot \frac{2}{3}\right)+(1-\alpha)\left(\frac{2}{3} \cdot \frac{2}{3}+\frac{1}{3} \cdot \frac{1}{3}\right)=\frac{5}{9}-\frac{1}{9} \alpha .
\end{aligned}
$$

As the probabilities of transitions to $w$ are equal from $t$ and $s$, we obtain that for all $k \in C(d)$, we have $\mathbb{E}_{t}^{y_{2}, y_{1}}(k)-\mathbb{E}_{s}^{x_{2}, a}(k)=0$, as desired.

For the third assertion, we consider a modified version of the game depicted in Figure 1, obtained by adding two new moves to player 2 at state $t$, namely $f^{\prime}$ and $g^{\prime}$. We define the transition probabilities of these new moves by

$$
\delta\left(t, *, f^{\prime}\right)=\delta(s, a, f) \quad \delta\left(t, *, g^{\prime}\right)=\delta(s, a, g) .
$$

To prove $\left[s \sqsubseteq_{1} t\right]>0$, we can proceed as in the proof of Theorem 3.7 noting that we can choose $y_{2}$ as in that proof (this is possible, as player 2 at $t$ has more moves available in the modified game). This leads to $\left[s \cong_{1} t\right]>0$.

To show that $\left[s \cong_{2} t\right]=0$, given the transition structure of the game, it suffices to show that $\left[s \sqsubseteq_{2} t\right]=0$ and $\left[t \sqsubseteq_{2} s\right]=0$. To show that $\left[s \sqsubseteq_{2} t\right]=0$, we show that for all $d$, we have:

$$
\forall x_{2} \in \mathcal{D}_{2}(s) . \exists y_{2} \in \mathcal{D}_{2}(t) . \forall y_{1} \in \mathcal{D}_{1}(t) . \forall k \in C(d) .\left(\mathbb{E}_{s}^{x_{2}, a}(k)-\mathbb{E}_{t}^{y_{2}, y_{1}}(k)\right)=0 .
$$

We can write any mixed move $x_{2} \in \mathcal{D}_{2}(s)$ as $x_{2}=\alpha f+(1-\alpha) g$. We can then choose $y_{2}=\alpha f^{\prime}+(1-\alpha) g^{\prime}$, and since at $t$ under $f^{\prime}, g^{\prime}$ the transition probabilities do not depend on the mixed move $y_{1}$ chosen by player 1 , we have that the transition probabilities from $s$ and $t$ match for all $0 \leq \alpha \leq 1$.

To show that $\left[t \sqsubseteq_{2} s\right]=0$, we need to show that:

$$
\forall y_{2} \in \mathcal{D}_{2}(t) . \exists x_{2} \in \mathcal{D}_{2}(s) . \exists y_{1} \in \mathcal{D}_{1}(t) . \forall k \in C(d) .\left(\mathbb{E}_{t}^{y_{2}, y_{1}}(k)-\mathbb{E}_{s}^{x_{2}, a}(k)\right)=0 .
$$

Any mixed move $y_{2} \in \mathcal{D}_{2}(t)$ can be written as

$$
y_{2}=\gamma[\alpha f+(1-\alpha) g]+(1-\gamma)\left[\beta f^{\prime}+(1-\beta) g^{\prime}\right],
$$

for some $\alpha, \beta, \gamma \in[0,1]$. We choose $x_{2}$ and $y_{1}$ as follows:

$$
\begin{aligned}
& x_{2}=\alpha \gamma\left[\frac{2}{3} f+\frac{1}{3} g\right]+(1-\alpha) \gamma\left[\frac{1}{3} f+\frac{2}{3} g\right]+(1-\gamma)[\beta f+(1-\beta) g] \\
& y_{1}=\alpha c+(1-\alpha) b .
\end{aligned}
$$

With these mixed moves, we have $\delta\left(s, a, x_{2}\right)=\delta\left(t, y_{1}, y_{2}\right)$, leading to the result. 
As a consequence of this theorem, we write $\left[\simeq_{g}\right]$ in place of $\left[\simeq_{1}\right]=\left[\simeq_{2}\right]$, to emphasize that the player 1 and player 2 versions of game equivalence metrics coincide.

3.5. Logical characterization of $\boldsymbol{a}$ priori metric. We now prove that $q \mu$ provides a logical characterization for the a priori metrics. We first state and prove two lemmas that lead to the desired result. The proof of the lemmas use ideas from [19] and [10]. We recall from Theorem 3.5 that we can compute $\left[\preceq_{1}\right]$ via Picard iteration, with $d_{n}=H_{\varliminf_{1}}^{n}(\mathbf{0})$ being the $n$-iterate.

We prove the existence of a logical characterization via a sequence of the following two lemmas. The first lemma proves that a priori metrics provide a bound for the difference in value of $q \mu$-formulas.

Lemma 3.9. The following assertions hold for all game structures.

(1) For all $\varphi \in q \mu_{1}^{+}$, and for all $s, t \in S$, we have $\llbracket \varphi \rrbracket(s)-\llbracket \varphi \rrbracket(t) \leq\left[s \preceq_{1} t\right]$.

(2) For all $\varphi \in q \mu$, and for all $s, t \in S$, we have $|\llbracket \varphi \rrbracket(s)-\llbracket \varphi \rrbracket(t)| \leq\left[s \simeq_{g} t\right]$.

Proof. We prove the first assertion. The proof is by induction on the structure of a (possibly open) formula $\varphi \in q \mu_{1}^{+}$. Call a variable valuation $\xi$ bounded if, for all variables $Z \in M$ Vars and states $s, t$, we have that $\xi(Z)(s)-\xi(Z)(t) \leq\left[s \preceq_{1} t\right]$. We prove by induction that for all $s, t \in S$, for all bounded variable valuations $\xi$, we have $\llbracket \varphi \rrbracket_{\xi}(s)-\llbracket \varphi \rrbracket_{\xi}(t) \leq\left[s \preceq_{1} t\right]$. For clarity, we sometimes omit writing the variable valuation $\xi$.

The base case for constants is trivial, and the case for observation variables follows since $[s \equiv t] \leq\left[s \preceq_{1} t\right]$. The case for variables $Z \in M$ Vars follows from the assumption of bounded variable valuations. For $\varphi_{1} \vee \varphi_{2}$, assume the induction hypothesis for $\varphi_{1}, \varphi_{2}$, and note that

$$
\begin{aligned}
\left(\llbracket \varphi_{1} \rrbracket(s) \sqcup \llbracket \varphi_{2} \rrbracket(s)\right)-\left(\llbracket \varphi_{1} \rrbracket(t)\right. & \left.\sqcup \llbracket \varphi_{2} \rrbracket(t)\right) \\
& \leq\left(\llbracket \varphi_{1} \rrbracket(s)-\llbracket \varphi_{1} \rrbracket(t)\right) \sqcup\left(\llbracket \varphi_{2} \rrbracket(s)-\llbracket \varphi_{2} \rrbracket(t)\right) \leq\left[s \preceq_{1} t\right] .
\end{aligned}
$$

The proof for $\wedge$ is similar. For $\varphi_{1} \oplus c$ and $\varphi_{1} \ominus c$, we have by induction hypothesis that $\llbracket \varphi_{1} \rrbracket(s)-\llbracket \varphi_{1} \rrbracket(t) \leq\left[s \preceq_{1} t\right]$, and so the "shifted versions" also satisfy the same bound.

For the induction step for pre $_{1}$, assume the induction hypothesis for $\varphi$, and note that we can choose $k \in C\left(\left[\preceq_{1}\right]\right)$ such that $k(s)=\llbracket \varphi \rrbracket(s)$ at all $s \in S$. We have, for all $s, t \in S$,

$$
\llbracket \operatorname{pre}_{1}(\varphi) \rrbracket(s)-\llbracket \operatorname{pre}_{1}(\varphi) \rrbracket(t) \leq \sup _{k \in C\left(\left[\preceq_{1}\right]\right)}\left(\operatorname{Pre}_{1}(k)(s)-\operatorname{Pre}_{1}(k)(t)\right) \leq\left[s \preceq_{1} t\right] .
$$

where the last inequality follows by noting that $\left[\preceq_{1}\right]$ is a fixpoint of $H_{\preceq_{1}}$.

The proof for the fixpoint operators is performed by considering their Picard iterates. We consider the case $\mu Z . \varphi$, the proof for $\nu Z . \varphi$ is similar. Let $\xi$ be a bounded variable valuation. Then, the variable valuation $\xi_{0}=\xi[Z \mapsto \mathbf{0}]$ is also bounded, and by induction hypothesis, the formula $\varphi$ when evaluated in the variable valuation $\xi_{0}$ satisfies

$$
\llbracket \varphi \rrbracket_{\xi_{0}}(s)-\llbracket \varphi \rrbracket_{\xi_{0}}(t) \leq\left[s \preceq_{1} t\right] .
$$

Now consider the variable valuation $\xi_{1}=\xi\left[Z \mapsto \llbracket \varphi \rrbracket \xi_{\xi_{0}}\right]$. From Equation (3.30), we get that $\xi_{1}$ is bounded, and again, by induction hypothesis, we have that $\llbracket \varphi \rrbracket_{\xi_{1}}(s)-\llbracket \varphi \rrbracket \xi_{1}(t) \leq\left[s \preceq_{1} t\right]$. In general, for $k \geq 0$, consider the variable valuation $\xi_{k+1}=\xi\left[Z \mapsto \llbracket \varphi \rrbracket \xi_{k}\right]$. By the above argument, each variable valuation $\xi_{k}$ is bounded, and so for every $k \geq 0$, we have

$$
\llbracket \varphi \rrbracket_{\xi_{k}}(s)-\llbracket \varphi \rrbracket_{\xi_{k}}(t) \leq\left[s \preceq_{1} t\right] .
$$


Taking the limit, as $k \rightarrow \infty$, we have that

$$
\lim _{k \rightarrow \infty}\left(\llbracket \varphi \rrbracket_{\xi_{k}}(s)-\llbracket \varphi \rrbracket_{\xi_{k}}(t)\right)=\llbracket \mu Z . \varphi \rrbracket_{\xi}(s)-\llbracket \mu Z . \varphi \rrbracket_{\xi}(t) \leq\left[s \preceq_{1} t\right] .
$$

The proof of the second assertion can be done along the same lines, using the symmetry of $\simeq_{g}$. The proof is again by induction on the structure of the formula. In particular, (3.29) can be proved for either player: for $n \geq 0$ and $i \in\{1,2\}$,

$$
\llbracket \operatorname{pre}_{i}(\varphi) \rrbracket(s)-\llbracket \operatorname{pre}_{i}(\varphi) \rrbracket(t) \leq \sup _{k \in C\left(\left[\simeq_{g}\right]\right)}\left(\operatorname{Pre}_{i}(k)(s)-\operatorname{Pre}_{i}(k)(t)\right) \leq\left[s \simeq_{g} t\right] .
$$

Negation can be dealt with by noting that $\llbracket \neg \varphi \rrbracket(s)-\llbracket \neg \varphi(t) \rrbracket=\llbracket \varphi \rrbracket(t)-\llbracket \varphi(s) \rrbracket$, and by using the symmetry of $\simeq_{g}$; the other cases are similar.

The second lemma states that the $q \mu$ formulas can attain the distance computed by the simulation metric.

Lemma 3.10. The following assertions hold for all game structures $G$, and for all states $s, t$ of $G$.

$$
\begin{aligned}
& {\left[s \preceq_{1} t\right] \leq \sup _{\varphi \in q \mu_{1}^{+}}(\llbracket \varphi \rrbracket(s)-\llbracket \varphi \rrbracket(t))} \\
& {\left[s \simeq_{g} t\right] \leq \sup _{\varphi \in q \mu}|\llbracket \varphi \rrbracket(s)-\llbracket \varphi \rrbracket(t)|}
\end{aligned}
$$

Proof. We show by induction on $n$ that $d_{n}(s, t) \leq \sup _{\varphi \in q \mu}(\llbracket \varphi \rrbracket(s)-\llbracket \varphi \rrbracket(t))$. The base case is trivial. For the induction step, the distance is:

$$
d_{i+1}(s, t)=\sup _{k \in C\left(d_{i}\right)}\left(\operatorname{Pre}_{1}(k)(s)-\operatorname{Pre}_{1}(k)(t)\right) .
$$

The challenge is to show that, for all $s, t \in S$, we can construct a formula $\psi_{s t}$ that witnesses the distance within an arbitrary $\varepsilon>0$ :

$$
d_{i+1}(s, t)-\varepsilon \leq \llbracket \psi_{s t} \rrbracket(s)-\llbracket \psi_{s t} \rrbracket(t) .
$$

To this end, let $k^{\star}$ be the value of $k$ that realizes the $\sup$ in (3.33) within $\varepsilon / 4$. By induction hypothesis, for each pair of states $s^{\prime}$ and $t^{\prime}$ we can choose $\varphi_{s^{\prime} t^{\prime}}^{\prime}$ such that

$$
d_{i}\left(s^{\prime}, t^{\prime}\right)-\varepsilon / 4 \leq \llbracket \varphi_{s^{\prime} t^{\prime}}^{\prime} \rrbracket\left(s^{\prime}\right)-\llbracket \varphi_{s^{\prime} t^{\prime}}^{\prime} \rrbracket\left(t^{\prime}\right) .
$$

Let $\varphi_{s^{\prime} t^{\prime}}$ be a shifted version of $\varphi_{s^{\prime} t^{\prime}}^{\prime}$, such that $\varphi_{s^{\prime} t^{\prime}}\left(s^{\prime}\right)=k^{\star}\left(s^{\prime}\right)$ :

$$
\varphi_{s^{\prime} t^{\prime}}=\varphi_{s^{\prime} t^{\prime}}^{\prime} \oplus\left(k^{\star}\left(s^{\prime}\right)-\llbracket \varphi_{s^{\prime} t^{\prime}}^{\prime} \rrbracket\left(s^{\prime}\right)\right) \text {. }
$$

We now prove that:

$$
\begin{aligned}
& \llbracket \varphi_{s^{\prime} t^{\prime}} \rrbracket\left(s^{\prime}\right)=k^{\star}\left(s^{\prime}\right) \\
& \llbracket \varphi_{s^{\prime} t^{\prime}} \rrbracket\left(t^{\prime}\right) \leq k^{\star}\left(t^{\prime}\right)+\varepsilon / 4 .
\end{aligned}
$$

Equality (3.37) is immediate from (3.36). We prove (3.38) as follows. We can rewrite (3.35) as

$$
\llbracket \varphi_{s^{\prime} t^{\prime}}^{\prime} \rrbracket\left(t^{\prime}\right)-\varepsilon / 4 \leq \llbracket \varphi_{s^{\prime} t^{\prime}}^{\prime} \rrbracket\left(s^{\prime}\right)-d_{i}\left(s^{\prime}, t^{\prime}\right) .
$$

Since $k^{\star} \in C\left(d_{i}\right)$, we have $k^{\star}\left(s^{\prime}\right)-k^{\star}\left(t^{\prime}\right) \leq d_{i}\left(s^{\prime}, t^{\prime}\right)$, or

$$
k^{\star}\left(t^{\prime}\right)-k^{\star}\left(s^{\prime}\right) \geq-d_{i}\left(s^{\prime}, t^{\prime}\right) \text {. }
$$


Plugging this relation into (3.39), we obtain

$$
\llbracket \varphi_{s^{\prime} t^{\prime}}^{\prime} \rrbracket\left(t^{\prime}\right)-\varepsilon / 4 \leq \llbracket \varphi_{s^{\prime} t^{\prime}}^{\prime} \rrbracket\left(s^{\prime}\right)+k^{\star}\left(t^{\prime}\right)-k^{\star}\left(s^{\prime}\right) .
$$

Plugging this relation into (3.36) evaluated at $t^{\prime}$, we obtain

$$
\llbracket \varphi_{s^{\prime} t^{\prime}} \rrbracket\left(t^{\prime}\right)-\varepsilon / 4 \leq \llbracket \varphi_{s^{\prime} t^{\prime}}^{\prime} \rrbracket\left(s^{\prime}\right)+k^{\star}\left(t^{\prime}\right)-k^{\star}\left(s^{\prime}\right) \oplus\left(k^{\star}\left(s^{\prime}\right)-\llbracket \varphi_{s^{\prime} t^{\prime}}^{\prime} \rrbracket\left(s^{\prime}\right)\right),
$$

or

$$
\llbracket \varphi_{s^{\prime} t^{\prime}} \rrbracket\left(t^{\prime}\right)-\varepsilon / 4 \leq k^{\star}\left(t^{\prime}\right)-\left(k^{\star}\left(s^{\prime}\right)-\llbracket \varphi_{s^{\prime} t^{\prime}}^{\prime} \rrbracket\left(s^{\prime}\right)\right) \oplus\left(k^{\star}\left(s^{\prime}\right)-\llbracket \varphi_{s^{\prime} t^{\prime}}^{\prime} \rrbracket\left(s^{\prime}\right)\right) \leq k^{\star}\left(t^{\prime}\right),
$$

which proves (3.38). Define now $\varphi_{s^{\prime}}=\bigwedge_{t^{\prime}} \varphi_{s^{\prime} t^{\prime}}$. From (3.37) and (3.38) we have

$$
\begin{aligned}
& \llbracket \varphi_{s^{\prime}} \rrbracket\left(s^{\prime}\right)=k^{\star}\left(s^{\prime}\right) \\
& \llbracket \varphi_{s^{\prime}} \rrbracket\left(t^{\prime}\right) \leq k^{\star}\left(t^{\prime}\right)+\varepsilon / 4 .
\end{aligned}
$$

Define then $\varphi=\bigvee_{s^{\prime}} \varphi_{s^{\prime}}$. From (3.42), (3.43), we have that

$$
k^{\star}\left(s^{\prime}\right) \leq \llbracket \varphi \rrbracket\left(s^{\prime}\right) \leq k^{\star}\left(s^{\prime}\right)+\varepsilon / 4 .
$$

for all $s^{\prime} \in S$. As formula $\psi_{s t}$, we propose thus to take the formula pre $(\varphi)$. From (3.44), we have that $\left|\llbracket \psi_{s t} \rrbracket(s)-\operatorname{Pre}_{1}\left(k^{\star}\right)(s)\right| \leq \varepsilon / 4$, and similarly, $\left|\llbracket \psi_{s t} \rrbracket(t)-\operatorname{Pre}_{1}\left(k^{\star}\right)(t)\right| \leq \varepsilon / 4$. By comparison with (3.33), and by the fact that $k^{\star}$ realizes the sup within $\varepsilon / 4$, we finally have (3.34), as desired.

From these two lemmas, we can conclude that $\llbracket q \mu \rrbracket$ provides a logical characterization for the a priori metrics, as stated by the next theorem.

Theorem 3.11. The following assertions hold for all game structures $G$, and for all states $s, t$ of $G$ :

$$
\left[s \preceq_{1} t\right]=\sup _{\varphi \in q \mu_{1}^{+}}(\llbracket \varphi \rrbracket(s)-\llbracket \varphi \rrbracket(t)) \quad\left[s \simeq_{g} t\right]=\sup _{\varphi \in q \mu}|\llbracket \varphi \rrbracket(s)-\llbracket \varphi \rrbracket(t)|
$$

We note that, due to Theorem 3.7, an analogous result does not hold for the a posteriori metrics. Together with the lack of reciprocity of the a posteriori metrics, this is a strong indication that the a priori metrics, and not the a posteriori ones, are the "natural" metrics on concurrent games.

Our metrics are not characterized by the probabilistic temporal logic PCTL [13, 3]. In fact, the values of PCTL formulas can change from true to false when certain probabilities cross given thresholds, so that PCTL formulas can have different boolean values on games that are very close in transition probabilities, and hence, very close in our metric. Quantitative metrics such as the ones developed in this paper are suited to quantitative-valued formulas, such as those of $q \mu$.

3.6. The Kernel. The kernel of the metric $\left[\simeq_{g}\right]$ defines an equivalence relation $\simeq_{g}$ on the states of a game structure: $s \simeq_{g} t$ iff $\left[s \simeq_{g} t\right]=0$. We call this the game bisimulation relation. Notice that by the reciprocity property of $\simeq_{g}$, the game bisimulation relation is canonical: $\simeq_{1}=\simeq_{2}=\simeq_{g}$. Similarly, we define the game simulation preorder $s \preceq_{1} t$ as the kernel of the directed metric $\left[\preceq_{1}\right]$, that is, $s \preceq_{1} t$ iff $\left[s \preceq_{1} t\right]=0$. Alternatively, it is possible to define $\preceq_{1}$ and $\simeq_{g}$ directly. Given a relation $R \subseteq S \times S$, let $B(R) \subseteq \mathcal{F}$ consist of all valuations $k \in \mathcal{F}$ such that, for all $s, t \in S$, if $s R t$ then $k(s) \leq k(t)$. We have the following result. 
Theorem 3.12. Given a game structure $G$, the relation $\preceq_{1}$ (resp. $\simeq_{1}$ ) can be characterized as the largest (resp. largest symmetrical) relation $R$ such that, for all states $s, t$ with sRt, we have $s \equiv t$ and

$$
\forall k \in B(R) . \forall x_{1} \in \mathcal{D}_{1}(s) . \exists y_{1} \in \mathcal{D}_{1}(t) . \forall y_{2} \in \mathcal{D}_{2}(t) . \exists x_{2} \in \mathcal{D}_{2}(s) .\left(\mathbb{E}_{t}^{y_{1}, y_{2}}(k) \geq \mathbb{E}_{s}^{x_{1}, x_{2}}(k)\right) .
$$

Proof. The proof proceeds by induction on the computation of the fixpoint relation $R$. We first present the case for $\preceq_{1}$. Call $R_{n}$ the $n$-th iterate of the simulation relation $R$, and let $d_{n}$ be the $n$-th iterate of $\left[\preceq_{1}\right]$, as in Theorem 3.5 . We prove by induction that, for all states $s, t \in S$, we have $s R_{n} t$ iff $d_{n}(s, t)=0$. We define $d_{0}(s, t)=[s \equiv t]$. The base case is then immediate because $s R_{0} t$ iff $d_{0}(s, t)=0$. Consider the induction step, for $n \geq 0$, and consider any states $s, t \in S$. Assume first that $d_{n+1}(s, t)>0$ : then, it is easy to show that we can find a value for $k$ in (3.45) that witnesses $(s, t) \notin R_{n+1}$, since the constraints on $k$ due to $B\left(R_{n}\right)$ are weaker than those due to $C\left(d_{n}\right)$. Conversely, assume that there is a $k \in B\left(R_{n}\right)$ that witnesses $(s, t) \notin R_{n+1}$. Then, by scaling all $k$ values so that they are all smaller than the smallest non-zero value of $d_{n}\left(s^{\prime}, t^{\prime}\right)$ for any $s^{\prime}, t^{\prime} \in S$, we can find a $k^{\prime} \in C\left(d_{n}\right)$ which also witnesses $d_{n+1}(s, t)>0$, as required.

The case for $\simeq_{g}$ is analogous, due to the similarity of the Picard iterations (3.14) for $\preceq_{1}$ and (3.15) for $\simeq_{g}$.

We note that the above theorem allows the computation of $\simeq_{g}$ via a partition-refinement scheme. From the logical characterization theorem, we obtain the following corollary.

Corollary 3.13. For any game structure $G$ and states $s, t$ of $G$, we have $s \simeq_{g}$ t iff $\llbracket \varphi \rrbracket(s)=$ $\llbracket \varphi \rrbracket(t)$ holds for every $\varphi \in q \mu$ and $s \preceq_{1} t$ iff $\llbracket \varphi \rrbracket(s) \leq \llbracket \varphi \rrbracket(t)$ holds for every $\varphi \in q \mu_{1}^{+}$.

3.7. Relation between Game Metrics and (Bi-)simulation Metrics. The a priori metrics assume an adversarial relationship between the players. We show that, on turnbased games, the a priori bisimulation metric coincides with the classical bisimulation metric where the players cooperate.

We define such "cooperative" simulation and bisimulation metrics $\left[\preceq_{12}\right]$ and $\left[\simeq_{12}\right]$ as the metric analog of classical (bi)simulation [22, 25]. We define the metric transformers $H_{\preceq_{12}}: \mathcal{M} \mapsto \mathcal{M}$ and $H_{\simeq_{12}}: \mathcal{M} \mapsto \mathcal{M}$, for all metrics $d \in \mathcal{M}$ and $s, t \in S$, by:

$$
\begin{aligned}
& H_{\preceq_{12}}(d)(s, t)=[s \equiv t] \sqcup \sup _{k \in C(d)} \sup _{x_{1} \in \mathcal{D}_{1}(s)} \sup _{x_{2} \in \mathcal{D}_{2}(s)} \inf _{y_{2} \in \mathcal{D}_{2}(t)} \inf _{y_{1} \in \mathcal{D}_{1}(t)}\left\{\mathbb{E}_{s}^{x_{1}, x_{2}}(k)-\mathbb{E}_{t}^{y_{1}, y_{2}}(k)\right\} . \\
& H_{\beth_{12}}(d)(s, t)=H_{\preceq_{12}}(d)(s, t) \sqcup H_{\preceq_{12}}(d)(t, s) .
\end{aligned}
$$

The metrics $\left[\preceq_{12}\right]$ and $\left[\simeq_{12}\right]$ are defined as the least fixed points of $H_{\preceq_{12}}$ and $H_{\simeq_{12}}$ respectively. The kernel of these metrics define the classical probabilistic simulation and bisimulation relations.

Theorem 3.14. The following assertions hold.

(1) On turn-based game structures, $\left[\simeq_{g}\right]=\left[\simeq_{12}\right]$.

(2) There is a deterministic game structure $G$ and states $s, t$ in $G$ such that $\left[s \simeq_{g} t\right]>$ $\left[s \simeq_{12} t\right]$.

(3) There is a deterministic game structure $G$ and states $s, t$ in $G$ such that $\left[s \simeq_{g} t\right]<$ $\left[s \simeq_{12} t\right]$. 


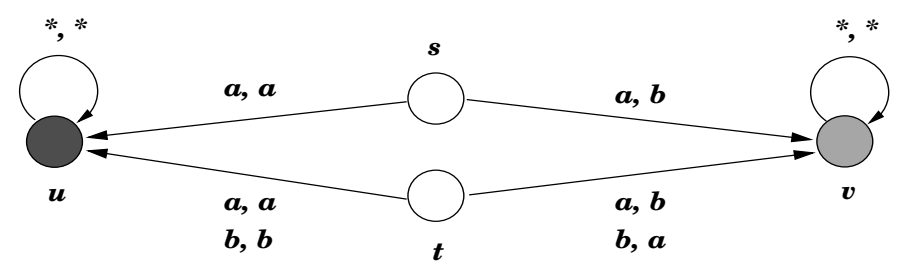

Figure $2:\left[s \simeq_{g} t\right]=\frac{1}{2}$ and $\left[s \simeq_{12} t\right]=0$

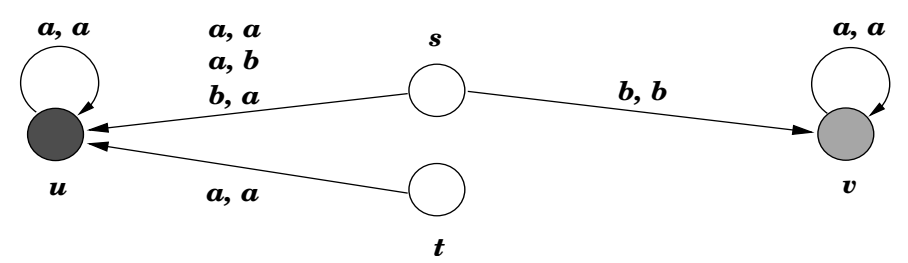

Figure 3: $\left[s \simeq_{g} t\right]=0$ but $\left[s \simeq_{12} t\right]=1$.

Proof. For the first part, since we have turn-based games, only one player has a choice of moves at each state. We say that a state $s$ belongs to player $i \in\{1,2\}$ if player $\sim i$ has only one move at $s$. First, notice that due to the presence of the variable turn, the metric distance between states belonging to different players is always 1, for all the metrics we consider. Thus, we focus on the metric distances between states belonging to the same player. Consider two player 1 states $s, t \in S$. From the definitions of $H_{\unlhd_{1}}$ and $H_{\unlhd_{12}}$, for $d \in \mathcal{M}$, by dropping the moves of player 2 , it is easy to see that $H_{\preceq_{1}}(d)=H_{\unlhd_{12}}(\bar{d})$, and $H_{\simeq_{g}}(d)=H_{\simeq_{12}}(d)$. Since this holds for any $d \in \mathcal{M}$, it holds for the fixpoints, $\left[\simeq_{g}\right]$ and $\left[\simeq_{12}\right]$.

The second part is proved by the game in Figure 2, where $[s \equiv t]=0$ and $[u \equiv v]=1$. The latter yields $\left[u \simeq_{g} v\right]=1$. Since player 1 has no choice of moves at state $s$, the maximum probability with which player 1 can guarantee a transition to either state $u$ or state $v$ is 0 . But from state $t$, by playing moves $a, b$ with probability $\frac{1}{2}$ each, player 1 can guarantee reaching states $u$ and $v$ with probability $\frac{1}{2}$, which implies that over all $k \in C(d)$, given that $d(u, v)=1$ from $\left[u \simeq_{g} v\right]=1$, the maximum $k$ expectation that player 1 can guarantee is $\frac{1}{2}$. Therefore $\left[s \simeq_{g} t\right]=\frac{1}{2}$. But if player 2 co-operates, then $\left[s \simeq_{12} t\right]=0$.

The third part is proved by the game in Figure 3 where again $[s \equiv t]=0$ and $[u \equiv v]=1$. Since the players don't have any moves to transition to state $v$ from state $t,\left[s \simeq_{12} t\right]=1$, whereas $\left[s \simeq_{g} t\right]=0$.

If we consider Markov decision processes (MDPs), we have that on $i$-MDPs, the metric $\preceq_{i}$ coincides with $\preceq_{12}$, since player $\sim i$ has no moves, for $i \in\{1,2\}$. On the other hand, the metric $\preceq_{\sim}$ provides no information on $\preceq_{12}$.

Theorem 3.15. The following assertions hold.

(1) For $i$-MDPs we have $\left[\preceq_{i}\right]=\left[\preceq_{12}\right]$.

(2) There is a deterministic 2-MDP $G$ with states $s, t$ such that $\left[s \preceq_{1} t\right]<\left[s \preceq_{12} t\right]$.

(3) There is a deterministic 2-MDP $G$ with states $s, t$ such that $\left[s \preceq_{1} t\right]>\left[s \preceq_{12} t\right]$.

Proof. From the definitions of $H_{\preceq_{1}}$ and $H_{\preceq_{12}}$, restricted to MDPs, where only one player has a choice of moves, the first assertion follows. 


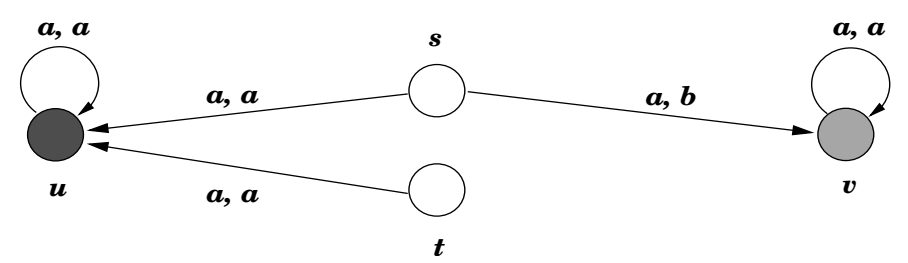

Figure 4: $\left[s \preceq_{1} t\right]=0$ and $\left[s \preceq_{12} t\right]=1$. Also, $\left[t \preceq_{1} s\right]=1$ and $\left[t \preceq_{12} s\right]=0$.

The second and third assertions are proved by the deterministic 2-MDP in Figure 4. where again $[s \equiv t]=0$ and $[u \equiv v]=1$. For the second assertion we note that since $d(u, v)=1$, for any choice of $k \in C(d)$, player 1 cannot get a higher expectation of $k$ from state $s$ when compared to state $t$, because at state $s$, player 2 always has a move that will lead to a state yielding a lower $k$ expectation. Therefore, $\left[s \preceq_{1} t\right]=0$. Further, for $k(v)=1$ and $k(u)=0$, which satisfies the constraints on $k$, we have no moves for either player from state $t$, which implies $\left[s \preceq_{12} t\right]=1$.

We prove the third assertion by showing that, for the 2-MDP of Figure 4, we have $\left[t \preceq_{1} s\right]>\left[\begin{array}{ll}t \preceq_{12} & s\end{array}\right]$ (which is the third assertion, with $s$ and $t$ exchanged). Note that when player 2 cooperates, the expectation of any $k \in C(d)$ from state $s$ is always at least as much as the expectation from state $t$. Thus $\left[t \preceq_{12} s\right]=0$. Finally, there exists a $k \in C(d)$, with $k(u)=1$ and $k(v)=0$, for which $\left[t \preceq_{1} s\right]=1$, which completes the proof.

3.8. Computation. We now show that the metrics are computable to any degree of precision. This follows since the definition of the distance between two states of a given game, as the least fixpoint of the metric transformer (3.9), can be written as a formula in the theory of reals, which is decidable [29]. Since the distance between two states may not be rational, we can only guarantee an approximate computation in general.

Without loss of generality, we assume that the states of $G$ are labeled $\left\{s_{1}, \ldots, s_{n}\right\}$ for some $n \in \mathbb{N}$. The construction is standard (see, e.g., [7]), we recapitulate the main steps. We denote by $\mathbf{R}$ the real-closed field $(\mathbb{R},+, \cdot, 0,1, \leq)$ of the reals with addition and multiplication. An atomic formula is an expression of the form $p>0$ or $p=0$ where $p$ is a (possibly) multi-variate polynomial with integer coefficients. An elementary formula is constructed from atomic formulas by the grammar

$$
\varphi::=a|\neg \varphi| \varphi \wedge \varphi|\varphi \vee \varphi| \exists x . \varphi \mid \forall x . \varphi
$$

where $a$ is an atomic formula, $\wedge$ denotes conjunction, $\vee$ denotes disjunction, $\neg$ denotes complementation, and $\exists$ and $\forall$ denote existential and universal quantification respectively. We write $\varphi \rightarrow \varphi^{\prime}$ as shorthand for $\neg \varphi \vee \varphi^{\prime}$. The semantics of elementary formulas are given in a standard way [4]. A variable $x$ is free in the formula $\varphi$ if it is not in the scope of a quantifier $\exists x$ or $\forall x$. An elementary sentence is a formula with no free variables. The theory of real-closed fields is decidable [29].

We introduce additional atomic formulas as syntactic sugar: for polynomials $p_{1}$ and $p_{2}$, we write $p_{1}=p_{2}$ for $p_{1}-p_{2}=0, p_{1}>p_{2}$ for $p_{1}-p_{2}>0$, and $p_{1} \geq p_{2}$ for $p_{1}-p_{2}=$ $0 \vee p_{1}-p_{2}>0$. Also, we write $p_{1} \leq p_{2}$ for $p_{2} \geq p_{1}$ and $p_{1}<p_{2}$ for $p_{2}>p_{1}$. Let $\vec{x}, \vec{y}$ denote vectors of variables, where the dimensions of the vectors will be clear from the context. For $\sim \in\{=, \leq, \geq\}$, we write $\vec{x} \sim \vec{y}$ for the pointwise ordering, that is, if $\bigwedge_{i} x_{i} \sim y_{i}$. A subset $C \subseteq \mathbb{R}^{m}$ is definable in $\mathbf{R}$ if there exists an elementary formula $\varphi_{C}(\vec{x})$ such that for any 
$\vec{x}_{0} \in \mathbb{R}^{m}$, we have $\varphi_{C}\left(\vec{x}_{0}\right)$ holds in $\mathbf{R}$ iff $\vec{x}_{0} \in C$. A function $f: \mathbb{R}^{k} \rightarrow \mathbb{R}^{m}$ is definable in $\mathbf{R}$ if there exists an elementary formula $\varphi_{f}(\vec{y}, \vec{x})$ with free variables $\vec{y}, \vec{x}$ such that for all constants $\vec{y}_{0} \in \mathbb{R}^{m}$ and $\vec{x}_{0} \in \mathbb{R}^{k}$ the formula $\varphi_{f}\left(\vec{y}_{0}, \vec{x}_{0}\right)$ is true in $\mathbf{R}$ iff $\vec{y}_{0}=f\left(\vec{x}_{0}\right)$. We start with some simple observations about definability.

Lemma 3.16. (a) If functions $f_{1}: \mathbb{R}^{k} \rightarrow \mathbb{R}^{m}$ and $f_{2}: \mathbb{R}^{k} \rightarrow \mathbb{R}^{m}$ are definable in $\mathbf{R}$ then so are the functions

$$
\begin{aligned}
& \left(f_{1}-f_{2}\right)(\vec{x})=f_{1}(\vec{x})-f_{2}(\vec{x}) \\
& \left(f_{1} \sqcup f_{2}\right)(\vec{x})=f_{1}(\vec{x}) \sqcup f_{2}(\vec{x})
\end{aligned}
$$

(b) If $f: \mathbb{R}^{k+l} \rightarrow \mathbb{R}^{m}$ is definable in $\mathbf{R}$, and $C \subseteq \mathbb{R}^{k}$ is definable in $\mathbf{R}$, then $\left.\sup _{C} f\right)$ : $\mathbb{R}^{l} \rightarrow \mathbb{R}^{m}$ defined as

$$
\left(\sup _{C} f\right)(\vec{y})=\sup _{\vec{x} \in C} f(\vec{x}, \vec{y})
$$

is definable in $\mathbf{R}$.

Proof. For part (a), let $\varphi_{1}(\vec{y}, \vec{x})$ and $\varphi_{2}(\vec{y}, \vec{x})$ be formulas defining $f_{1}$ and $f_{2}$ respectively. Then, $f_{1}-f_{2}$ is defined by the formula

$$
\exists \vec{z}_{1} \cdot \exists \vec{z}_{2} \cdot\left(\varphi_{1}\left(\vec{z}_{1}, \vec{x}\right) \wedge \varphi_{2}\left(\vec{z}_{2}, \vec{x}\right) \wedge \vec{y}=\vec{z}_{1}-\vec{z}_{2}\right),
$$

and $f_{1} \sqcup f_{2}$ is defined by the formula

$$
\exists \vec{z}_{1} \cdot \exists \vec{z}_{2} \cdot\left(\varphi_{1}\left(\vec{z}_{1}, \vec{x}\right) \wedge \varphi_{2}\left(\vec{z}_{2}, \vec{x}\right) \wedge \bigwedge_{i}\left[\left(\vec{z}_{1, i} \geq \vec{z}_{2, i} \wedge \vec{y}_{i}=\vec{z}_{1, i}\right) \vee\left(\vec{z}_{1, i}<\vec{z}_{2, i} \wedge \vec{y}_{i}=\vec{z}_{2, i}\right)\right]\right) .
$$

For part (b), let $\varphi_{f}(\vec{z}, \vec{x}, \vec{y})$ define $f$, where $\vec{x}$ is of dimension $k, \vec{y}$ of dimension $l$, and $\vec{z}$ of dimension $m$, respectively. Let $\psi_{C}(\vec{x})$ define $C$. Then, the following formula with free variables $\vec{z}, \vec{y}$ (call it $\varphi(\vec{z}, \vec{y})$ ) states that $\vec{z}$ is an upper bound of $f(\vec{x}, \vec{y})$ for all $\vec{x} \in C$ :

$$
\forall \vec{x}_{1} \cdot \forall \vec{z}_{1} \cdot\left(\psi_{C}\left(\vec{x}_{1}\right) \wedge \varphi_{f}\left(\vec{z}_{1}, \vec{x}_{1}, \vec{y}\right) \rightarrow \vec{z}_{1} \leq \vec{z}\right),
$$

and $\sup _{C} f$ is defined by the formula with free variables $\vec{z}, \vec{y}$ given by:

$$
\varphi(\vec{z}, \vec{y}) \wedge \forall \vec{z}_{1} \cdot\left(\varphi\left(\vec{z}_{1}, \vec{y}\right) \rightarrow \vec{z} \leq \vec{z}_{1}\right) .
$$

Theorem 3.17. Let $G$ be a game structure and $s, t$ states of $G$. For all rationals $v$, and all $\epsilon>0$, it is decidable if $\left|\left[s \preceq_{1} t\right]-v\right|<\epsilon$ and if $\left|\left[s \simeq_{g} t\right]-v\right|<\epsilon$. It is decidable if $s \preceq_{1} t$ and if $s \simeq_{g} t$.

Proof. First, we use a result of Weyl [33] that the minmax value of a matrix game with payoffs in $\mathbb{R}$ can be written as an elementary formula in the theory of real-closed fields. This implies that for any state $s$, the function $\operatorname{Pre}_{1}(\vec{k})(s)$ is definable in $\mathbf{R}$. Also, for $d \in \mathcal{M}$, the set $C(d)$ is definable in $\mathbf{R}$ (since conjunctions of linear constraints are definable in $\mathbf{R}$ ). Hence, by Lemma 3.16 (a) and (b), we have that $\sup _{\vec{k} \in C(d)}\left(\operatorname{Pre}_{1}(\vec{k})(s)-\operatorname{Pre}_{1}(\vec{k})(t)\right)$ is definable for any metric $d \in \mathcal{M}$, and states $s$ and $t$ of $G$. By another application of Lemma 3.16(a), we have that the function

$$
H_{\preceq_{1}}(d)(s, t)=(s \equiv t) \sqcup \sup _{\vec{k} \in C(d)}\left(\operatorname{Pre}_{1}\left(\vec{k}(s)-\operatorname{Pre}_{1}(\vec{k})(t)\right) .\right.
$$

is definable for $d \in \mathcal{M}$ and states $s$ and $t$ of $G$.

Consider the set of free variables $\{y(s, t), d(s, t) \mid s, t \in S\}$, where $d$ is a vector of $n^{2}$ free variables defining the metric $d$, and where $y$ is a vector of $n^{2}$ variables. Let $\varphi(y, d)$ be 
a formula in $\mathbf{R}$, with free variables in the above set, such that $\varphi(y, d)$ is true iff $y(s, t)=$ $H_{\preceq_{1}}(d)(s, t)$ holds for all $s, t \in S$. Then the formula $\varphi^{*}(y)$ with free variables $y$, defined as:

$$
\exists d .(\varphi(y, d) \wedge y=d),
$$

defines a fixpoint of $H_{\preceq_{1}}(d)$. Finally, the formula $\psi(y)$, given by

$$
\varphi^{*}(y) \wedge \forall y^{\prime} \cdot\left(\varphi^{*}\left(y^{\prime}\right) \rightarrow y \leq y^{\prime}\right) .
$$

defines the least fixpoint of $H_{\preceq_{1}}$ (again, $y^{\prime}=\left\{y^{\prime}(s, t) \mid s, t \in S\right\}$ is a matrix of $n^{2}$ variables, and $y \leq y^{\prime}$ iff $y(s, t) \leq y^{\prime}(s, t)$ for all $\left.s, t \in S\right)$. Thus, $\psi(y)$ is true iff $y(s, t)=\left[s \preceq_{1} t\right]$ for all $s, t \in S$.

While this shows that $\left[s \preceq_{1} t\right]$ is algebraic, there are game structures $G$ with all transition probabilities being rational, but with states $s$ and $t$ of $G$ such that $\left[s \preceq_{1} t\right]$ is irrational. So, we use the formula above to approximate the value of $\left[s \preceq_{1} t\right]$ to within a constant $\epsilon$. For states $s, t$ and rationals $v, \epsilon$, we have that $\left|\left[s \preceq_{1} t\right]-v\right|<\epsilon$ iff $\exists y .(\psi(y) \wedge|y(s, t)-v|<\epsilon)$ is valid, and this can be decided since $\mathbf{R}$ is decidable.

A similar construction shows that the question whether $\left|\left[s \simeq_{g} t\right]-v\right|<\epsilon$, is decidable for states $s, t$ and rationals $v, \epsilon$ : we ensure that $y$ is a symmetric fixpoint by conjoining to $\varphi^{*}(y)$ constraints $y(s, t)=y(t, s)$ for all states $s, t$.

If the formula $\exists y \cdot(\psi(y) \wedge y(s, t)=0)$, where we assert that the distance between $s$ and $t$ is zero, is valid, we can conclude that $s \preceq_{1} t$. This implies that the relation $s \preceq_{1} t$ is decidable for any game structure $G$ and states $s$ and $t$ of $G$. A similar construction for $\simeq_{g}$ shows that the relation $s \simeq_{g} t$ is also decidable for any game structure $G$ and states $s, t$ of $G$.

\section{Discussion}

Our derivation of $\preceq_{i}$ and $\simeq_{g}$, for $i \in\{1,2\}$, as kernels of metrics, seems somewhat abstruse: most equivalence or similarity relations have been defined, after all, without resorting to metrics. We now point out how a generalization of the usual definitions [25, 2, 9, 10], suggested in [6, 19], fails to produce the "right" relations. Furthermore, the flawed relations obtained as a generalization of [25, 2, 9, 10] are no simpler than our definitions, based on kernel metrics. Thus, our study of game relations as kernels of metrics carries no drawbacks in terms of leading to more complicated definitions. Indeed, we believe that the metric approach is the superior one for the study of game relations.

We outline the flawed generalization of [25, 2, 9, 10] as proposed in [6, 19], explaining why it would seem a natural generalization. The alternating simulation of 2 is defined over deterministic game structures. Player- $i$ alternating simulation, for $i \in\{1,2\}$, is the largest relation $R$ satisfying the following conditions, for all states $s, t \in S: s R t$ implies $s \equiv t$ and $\forall x_{i} \in \Gamma_{i}(s) . \exists y_{i} \in \Gamma_{i}(t) . \forall y_{\sim i} \in \Gamma_{\sim i}(t) . \exists x_{\sim i} \in \Gamma_{\sim i}(s) . \tau\left(s, x_{1}, x_{2}\right) R \tau\left(t, y_{1}, y_{2}\right)$.

The MDP relations of [25], later extended to metrics by [9, 10, rely on the fixpoint (3.2), where sup plays the role of $\forall$, inf plays the role of $\exists$, and $R$ is replaced by distribution equality modulo $R$, or $\sqsubseteq_{R}$. This strongly suggests — incorrectly - that equivalences for general games (probabilistic, concurrent games) can be obtained by taking the double quantifier alternation $\forall \exists \forall \exists$ in the definition of alternating simulation, changing all $\forall$ into sup, all $\exists$ into inf, and replacing $R$ by $\sqsubseteq_{R}$. The definition that would result is as follows. We parametrize the new relations by a player $i \in\{1,2\}$, as well as by whether mixed moves 
or only pure moves are allowed. For a relation $R \subseteq S \times S$, for $M \in\{\Gamma, \mathcal{D}\}$, for all $s, t \in S$ and $i \in\{1,2\}$ consider the following conditions:

- (loc) $s R t$ implies $s \equiv t$.

- $(M$-i-altsim) $s R t$ implies $\forall x_{i} \in M_{i}(s) . \exists y_{i} \in M_{i}(t) . \forall y_{\sim i} \in M_{\sim i}(t) . \exists x_{\sim i} \in M_{\sim i}(s) . \delta\left(s, x_{1}, x_{2}\right) \sqsubseteq_{R} \delta\left(t, y_{1}, y_{2}\right) ;$

We then define the following relations:

- For $i \in\{1,2\}$ and $M \in\{\Gamma, \mathcal{D}\}$, player-i $M$-alternating simulation $\sqsubseteq_{i}^{M}$ is the largest relation that satisfies (loc) and ( $M$-i-altsim).

- For $i \in\{1,2\}$ and $M \in\{\Gamma, \mathcal{D}\}$, player- $i M$-alternating bisimulation $\cong_{i}^{M}$ is the largest symmetrical relation that satisfies (loc) and ( $M$-i-altsim).

Over deterministic game structures, the definitions of $\complement_{i}^{\Gamma}$ and $\cong_{i}^{\Gamma}$ coincide with the alternating simulation and bisimulation relations of [2]. In fact, $\bigsqcup_{i}^{\Gamma}$ and $\cong_{i}^{\Gamma}$ capture the deterministic semantics of $q \mu$, and thus in some sense generalize the results of [2] to probabilistic game structures.

Theorem 4.1. For any game structure $G$ and states $s, t$ of $G$, the following assertions hold: (1) $s \cong{ }_{i} t$ iff $\llbracket \varphi \rrbracket^{\Gamma}(s)=\llbracket \varphi \rrbracket^{\Gamma}(t)$ holds for every $\varphi \in q \mu_{i}$.

(2) $s \bigsqcup_{i}^{\Gamma} t$ iff $\llbracket \varphi \rrbracket^{\Gamma}(s) \leq \llbracket \varphi \rrbracket^{\Gamma}(t)$ holds for every $\varphi \in q \mu_{i}^{+}$.

The following lemma states that $\complement_{i}^{\mathcal{D}}$ and $\cong_{i}^{\mathcal{D}}$ are the kernels of $\left[\sqsubseteq_{i}\right]$ and $\left[\cong_{i}\right]$, connecting thus the result of combining the definitions of [25] and [2] with a posteriori metrics.

Lemma 4.2. For all game structures $G$, all players $i \in\{1,2\}$, and all states $s, t$ of $G$, we have $s \sqsubseteq_{i}^{\mathcal{D}} t$ iff $\left[s \sqsubseteq_{i} t\right]=0$, and $s \cong_{i}^{\mathcal{D}} t$ iff $\left[s \cong_{i} t\right]=0$.

We are now in a position to prove that neither the $\Gamma$-relations not the $\mathcal{D}$-relations are the "canonical" relations on general concurrent games, since neither characterizes $\llbracket q \mu \rrbracket$. In particular, the $\mathcal{D}$-relations are too fine, and the $\Gamma$-relations are incomparable with the relations $\preceq_{i}$ and $\simeq_{g}$, for $i \in\{1,2\}$. We prove these negative results first for the $\mathcal{D}$-relations. They follow from Theorem 3.7 and 3.11 .

Theorem 4.3. The following assertions hold:

(1) For all game structures $G$, all states $s, t$ of $G$, and all $i \in\{1,2\}$, we have that $s \sqsubseteq_{i}^{\mathcal{D}} t$ implies $s \preceq_{i} t$, and $s \cong_{i}^{\mathcal{D}} t$ implies $s \simeq_{i} t$.

(2) There is a game structure $G$, and states $s, t$ of $G$, such that $s \preceq_{i} t$ but $s \nsubseteq_{i}^{\mathcal{D}} t$.

(3) There is a game structure $G$, and states $s, t$ of $G$, such that $\llbracket \varphi \rrbracket(s)=\llbracket \varphi \rrbracket(t)$ for all $\varphi \in q \mu$, but $s \varlimsup_{i}^{\mathcal{D}} t$ for some $i \in\{1,2\}$.

We now turn our attention to the $\Gamma$-relations, showing that they are incomparable with $\preceq_{i}$ and $\simeq_{g}$, for $i \in\{1,2\}$.

Theorem 4.4. The following assertions hold:

(1) There exists a deterministic game structure $G$ and states $s, t$ of $G$ such that $s \sqsubseteq_{1}^{\Gamma} t$ but $s \swarrow_{1} t$, and $s \cong_{1}^{\Gamma} t$ but $s \varkappa_{g} t$.

(2) There exists a turn-based game structure $G$ and states $s, t$ of $G$ such that $s \preceq_{1} t$ but $s \nsubseteq_{1}^{\Gamma} t$. and $s \simeq_{g} t$ but $s \varlimsup_{1}^{\Gamma} t$.

Proof. The first assertion is proved via the deterministic game in Figure 5, where $[s \equiv t]=0$ and $[u \equiv v]=1$ and $\Gamma_{1}(s)=\Gamma_{2}(s)=\{a, b\}$ and $\Gamma_{1}(t)=\Gamma_{2}(t)=\{a, b, c\}$. In the figure, we use the variables $x$ and $y$ to represent moves: if player 1 and player 2 moves coincide, $u$ is 


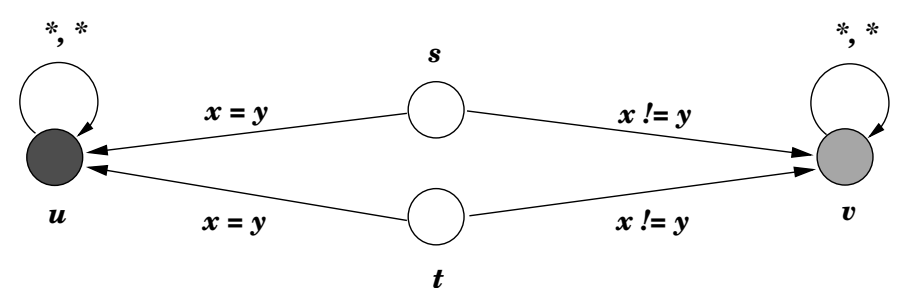

Figure 5: $s \sqsubseteq_{1}^{\Gamma} t$ but $s \swarrow_{1} t$ and $s \cong_{1}^{\Gamma} t$ but $s \varkappa_{g} t$

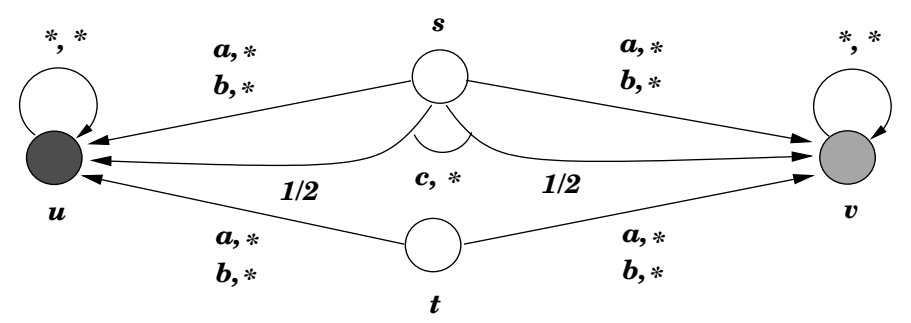

Figure 6: $s \preceq_{1} t$ but $s \nsubseteq_{1}^{\Gamma} t$ and $s \simeq_{g} t$ but $s \neq_{1}^{\Gamma} t$.

the successor state, otherwise it is $v$. Thus, the game from $s$ is the usual "penny-matching" game; the game from $t$ is a version of "penny-matching" with 3 -sided pennies.

It can be seen that $s \sqsubseteq_{1}^{\Gamma} t$. On the other hand, we have $s \swarrow_{1} t$. Indeed, from state $s$, by playing both $a$ and $b$ with probability $\frac{1}{2}$, player 1 can ensure that the probability of a transition to $u$ is $\frac{1}{2}$. On the other hand, from state $t$, player 1 can achieve at most probability $\frac{1}{3}$ of reaching $u$ (this maximal probability is achieved by playing all of $a, b, c$ with probability $\frac{1}{3}$ ). The result then follows using Theorem 3.11 .

The second assertion is proved via the game in Figure 6. We have $s \Xi_{1}^{\Gamma} t$ : clearly, player-1's move $c$ at state $s$ cannot be mimicked at $t$ when the game is restricted to pure moves. On the other hand, we have $s \preceq_{1} t$ : since the move $c$ at $s$ can be imitated via the mixed move that plays both $a$ and $b$ at $t$ with probability $\frac{1}{2}$ each, all $q \mu$ formulas have the same value, under $\llbracket \cdot \rrbracket$, at $s$ and $t$, and the result follows once more using Theorem 3.11 .

Finally, we remark that, in view of Theorem 3.12 , the definitions of the relations $\preceq_{i}$ and $\simeq_{g}$ for $i \in\{1,2\}$ are no more complex than the definitions of $\complement_{1}^{\mathcal{D}}, \sqsubseteq_{1}^{\Gamma}, \cong_{1}^{\mathcal{D}}$, and $\cong_{1}^{\Gamma}$.

\section{Conclusions}

We have introduced the metrics and relations that constitute the natural generalizations of simulation and bisimulation to stochastic games on graphs. These relations and metrics are tight, in the sense that the distance between two states is equal to the maximum difference in value that properties of the quantitative $\mu$-calculus can assume at the two states: in other words, the relations characterize quantitative $\mu$-calculus, in the same way in which ordinary bisimulation characterizes $\mu$-calculus. The paper also provided a full picture of the connection between the new metrics and relations, and the relations previously considered for games. 
The main point left open by the paper concerns the algorithms for the computation of the relations and metrics. The algorithms we provided rely on the decidability of the theory of reals; it is an open question whether more efficient, and more direct, algorithms exist, for the metrics or at least for the relations.

Acknowledgments. The first author was supported in part by the National Science Foundation grants CNS-0720884 and CCR-0132780. The second author was supported in part by the National Science Foundation grants CCF-0427202, CCF-0546170. The fourth author was supported in part by the Netherlands Organization for Scientific Research grant 642.000.505 and the EU grants IST-004527 and FP7-ICT-2007-1 214755.

\section{REFERENCES}

[1] R. Alur, T.A. Henzinger, and O. Kupferman. Alternating time temporal logic. J. ACM, 49:672-713, 2002.

[2] R. Alur, T.A. Henzinger, O. Kupferman, and M.Y. Vardi. Alternating refinement relations. In CONCUR 98: Concurrency Theory. 9th Int. Conf., volume 1466 of Lect. Notes in Comp. Sci., pages 163-178. Springer-Verlag, 1998.

[3] A. Aziz, V. Singhal, F. Balarin, R.K. Brayton, and A.L. Sangiovanni-Vincentelli. It usually works: The temporal logic of stochastic systems. In Computer Aided Verification, volume 939 of Lect. Notes in Comp. Sci. Springer-Verlag, 1995.

[4] C. C. Chang and H. J. Keisler. Model theory. Studies in Logic and the Foundations of Mathematics. North-Holland, Amsterdam., 1973.

[5] L. de Alfaro, T.A. Henzinger, and O. Kupferman. Concurrent reachability games. In Proc. 39th IEEE Symp. Found. of Comp. Sci., pages 564-575. IEEE Computer Society Press, 1998.

[6] L. de Alfaro, T.A. Henzinger, and R. Majumdar. Discounting the future in systems theory. In Proc. 30th Int. Colloq. Aut. Lang. Prog., volume 2719 of Lect. Notes in Comp. Sci., pages 1022-1037. SpringerVerlag, 2003.

[7] L. de Alfaro and R. Majumdar. Quantitative solution of omega-regular games. Journal of Computer and System Sciences, 68:374-397, 2004.

[8] C. Derman. Finite State Markovian Decision Processes. Academic Press, 1970.

[9] J. Desharnais, V. Gupta, R. Jagadeesan, and P. Panangaden. Metrics for labelled markov systems. In CONCUR 99: Concurrency Theory. 10th Int. Conf., volume 1664 of Lect. Notes in Comp. Sci., pages 258-273. Springer, 1999.

[10] J. Desharnais, V. Gupta, R. Jagadeesan, and P. Panangaden. Approximating labelled markov processes. Information and Computation, 2002.

[11] J. Desharnais, V. Gupta, R. Jagadeesan, and P. Panangaden. The metric analogue of weak bisimulation for probabilistic processes. In Proc. 17th IEEE Symp. Logic in Comp. Sci., pages 413-422, 2002.

[12] J. Filar and K. Vrieze. Competitive Markov Decision Processes. Springer-Verlag, 1997.

[13] H. Hansson and B. Jonsson. A logic for reasoning about time and reliability. Formal Asp. Comput., 6(5):512-535, 1994.

[14] C.-C. Jou and S.A. Smolka. Equivalences, congruences and complete axiomatizations for probabilistic processes. In CONCUR 90: Concurrency Theory. 1st Int. Conf., volume 458 of Lect. Notes in Comp. Sci., pages 367-383. Springer-Verlag, 1990.

[15] J.G. Kemeny, J.L. Snell, and A.W. Knapp. Denumerable Markov Chains. D. Van Nostrand Company, 1966.

[16] D. Kozen. A probabilistic PDL. In Proc. 15th ACM Symp. Theory of Comp., pages 291-297, 1983.

[17] D. Kozen. Results on the propositional $\mu$-calculus. Theoretical Computer Science, 27(3):333-354, 1983.

[18] K.G. Larsen and A. Skou. Compositional verification of probabilistic processes. In W.R. Cleaveland, editor, CONCUR 92: Concurrency Theory. 3rd Int. Conf., volume 630 of Lect. Notes in Comp. Sci. Springer-Verlag, 1992.

[19] R. Majumdar. Symbolic algorithms for verification and control. PhD thesis, University of California, Berkeley, 2003. 
[20] D.A. Martin. The determinacy of Blackwell games. The Journal of Symbolic Logic, 63(4):1565-1581, 1998.

[21] A. McIver and C. Morgan. Abstraction, Refinement, and Proof for Probabilistic Systems. Monographs in Computer Science. Springer-Verlag, 2004.

[22] R. Milner. Operational and algebraic semantics of concurrent processes. In J. van Leeuwen, editor, Handbook of Theoretical Computer Science, volume B, pages 1202-1242. Elsevier Science Publishers (North-Holland), Amsterdam, 1990.

[23] M.J. Osborne and A. Rubinstein. A Course in Game Theory. MIT Press, 1994.

[24] R. Segala. Modeling and Verification of Randomized Distributed Real-Time Systems. PhD thesis, MIT, 1995. Technical Report MIT/LCS/TR-676.

[25] R. Segala and N.A. Lynch. Probabilistic simulations for probabilistic processes. In CONCUR 94: Concurrency Theory. 5th Int. Conf., volume 836 of Lect. Notes in Comp. Sci., pages 481-496. SpringerVerlag, 1994.

[26] R. Segala and N.A. Lynch. Probabilistic simulations for probabilistic processes. Nordic Journal of Computing, 2(2):250-273, 1995.

[27] L.S. Shapley. Stochastic games. Proc. Nat. Acad. Sci. USA, 39:1095-1100, 1953.

[28] M. Sion. On general minimax theorems. Pacific Journal of Mathematics., 8:171-176, 1958.

[29] A. Tarski. A Decision Method for Elementary Algebra and Geometry. University of California Press, Berkeley and Los Angeles, 1951.

[30] F. van Breugel and J. Worrel. An algorithm for quantitative verification of probabilistic transition systems. In CONCUR 01: Concurrency Theory. 12th Int. Conf., volume 2154 of Lect. Notes in Comp. Sci., pages 336-350, 2001.

[31] F. van Breugel and J. Worrel. Towards quantitative verification of probabilistic systems. In Proc. 28th Int. Colloq. Aut. Lang. Prog., volume 2076 of Lect. Notes in Comp. Sci., pages 421-432. Springer-Verlag, 2001.

[32] J. von Neumann and O. Morgenstern. Theory of Games and Economic Behavior. New York: John Wiley and Sons, 1944.

[33] H. Weyl. Elementary proof of a minmax theorem due to von Neumann. In Contributions to the Theory of Games, I, volume 24 of Annals of Mathematical Studies, pages 19-25. Princeton University Press, 1950. 